\title{
DIVERSIDAD DE ARAÑAS (ARANEAE, ARANEOMORPHAE) EN LA SELVA DE MONTAÑA: UN CASO DE ESTUDIO EN LAS YUNGAS ARGENTINAS
}

\author{
Gonzalo D. Rubio
}

Consejo Nacional de Investigaciones Científicas y Técnicas (CONICET), Instituto de Biología Subtropical, Universidad Nacional de Misiones (IBS, UNaM), Puerto Iguazú, Misiones, Argentina. E-mail: grubio@conicet.gov.ar

\section{RESUMEN}

Se estudia la diversidad de arañas de vegetación de las yungas del noroeste argentino, integrando dos escalas: local (diversidad $\alpha$, estructura de comunidades) y su proyección a diversidad regional (diversidad $\beta$ ). Se muestrearon 26 sitios en la provincia de Salta, representando diferentes ambientes/pisos altitudinales de yungas sensu stricto ( $\mathrm{SP}=$ selva pedemontana, $\mathrm{SM}=$ selva montana, $\mathrm{BM}=$ bosque montano), yungas sensu lato ( $\mathrm{C}$-s $=$ conectividad entre centro y sur de yungas, $\mathrm{YT}=$ yungas en transición) y sitios de Chaco Serrano (ChS) como contraste. Se realizaron muestreos estacionales durante un año, tomando 10 muestras con G-Vac (aspirador entomológico) sobre vegetación. Se obtuvo un total de 6412 ejemplares, representando 188 especies y 34 familias (sólo yungas). Theridiidae, Anyphaenidae y Linyphiidae fueron dominantes. La mayor riqueza correspondió a Araneidae, Salticidae y Theridiidae. Especies dominantes fueron Chibchea salta (Pholcidae), Dubiaranea msp111 (Linyphiidae) y Mysmena msp110 (Mysmenidae). Diferencias relevantes en composición y abundancia separan dos grupos de ambientes: (Cc-s+SP+YT+ChS) vs. (SM+BM). Dictynidae, Oxyopidae y Philodromidae se asocian a pisos de menor altitud (Cc-s, YT, ChS). Los ambientes SP y YT contienen la mayor riqueza especifica y diversidad, mientras que SM y BM presentaron la mayor similitud. En Cc-s y ChS se observaron las mayores diferencias con los demás ambientes, excepto SP. La complementariedad y coeficientes e índices de similitud revelaron alta diversidad $\beta$ en la región. En consecuencia, se sugiere que además de reforzar la protección en los pisos transicionales de yungas (hábitats más deteriorados y diversos para las arañas), la gestión de conservación debería estar orientada en toda el área a promover la heterogeneidad espacial natural de las Yungas, haciendo especial hincapié en el mosaico de hábitats que constituyen cada estrato diferente.

Palabras clave: Araneae; diversidad de arañas; ecología de comunidades; Yungas.

\section{ABSTRACT}

Spider diversification (Araneae, Araneomorphae) in mountain rainforest: a case study in the Argentine Yungas

The spider diversity from yungas vegetation in northwestern Argentina is studied, integrating two levels: local ( $\alpha$ diversity, community structures) and a projection at regional level of diversity ( $\beta$ diversity). Twenty six sites in Salta Province were sampled, representing different ambient/altitudinal strata of yungas sensu stricto $(\mathrm{SP}=$ pedemontane rainforest, $\mathrm{SM}=$ montane rainforest and $\mathrm{BM}=$ montane forest), yungas sensu lato $(\mathrm{C} c-s=$ yungas central and southern sectors connectivity areas, $\mathrm{YT}=$ transitional yungas), and Chaco Serrano sites (ChS) as contrast. The sampling was carried out seasonally for one year taking 10 samples of vegetation with G-Vac method. A total of 6412 spiders, 188 species and 34 families were obtained (only yungas). Theridiidae, Anyphaenidae and Linyphiidae were dominant. The highest richness was observed in Araneidae, Salticidae and Theridiidae. Chibchea salta (Pholcidae), Dubiaranea msp111 (Linyphiidae) and Mysmena msp110 (Mysmenidae) were dominant species. Relevant differences in species composition and abundance highlighted two groups of environment ( $\mathrm{Cc}-\mathrm{s}+\mathrm{SP}+\mathrm{YT}+\mathrm{ChS})$ vs. $(\mathrm{SM}+\mathrm{BM})$. Dictynidae, Oxyopidae and Philodromidae are associated with lower altitudinal floors (Cc-s, YT, ChS). The greatest species richness and diversity were recorded in SP and YT. The highest similarity was recorded in SM and BM; the major differences were observed in $\mathrm{Cc}-\mathrm{s}$ and $\mathrm{ChS}$ compared with the other ambient, except with SP. Complementarity and similarity indices and coefficients revealed high $\beta$ diversity in the region. Thus, it is suggested that besides reinforcing protection in transitional levels Yungas (the most disturbed and diverse habitats for spiders), conservation management in the area should be directed towards promoting natural spatial heterogeneity of Yungas, giving special emphasis to habitat mosaics that constitute each different stratum.

Key words: Araneae; community ecology; spider diversity; Yungas. 
Recibido/Received: 18/03/2015; Aceptado/Accepted: 19/06/2015; Publicado en linea/Published online: 19/08/2015

Cómo citar este artículo/Citation: Rubio, G. D. 2015. Diversidad de arañas (Araneae, Araneomorphae) en la selva de montaña: un caso de estudio en las yungas argentinas. Grael/sia, 71(2): e029. http://dx.doi.org/10.3989/graellsia.2015. v71.134

Copyright: (c) 2015 SAM y CSIC. Salvo indicación contraria, todos los contenidos de la edición electrónica de Grael/sia se distribuyen bajo licencia de uso y distribución Creative Commons Reconocimiento no Comercial 3.0. España (cc-by-nc).

\section{Introducción}

Las araneomorfas (Araneae, Araneomorphae) han ganado una amplia aceptación en los estudios ecológicos como indicadores de unidades ambientales (Clausen, 1986; Maelfert et al., 1990). Se trata de un grupo faunístico ampliamente distribuido en todos los ecosistemas terrestres, presente incluso en ambientes dulceacuícolas (Turnbull, 1973). Las arañas son particularmente diversas en las selvas tropicales y subtropicales, donde se entiende que existen en mayor proporción, estimada en 170000 especies, y se considera que alrededor del $80 \%$ de la araneofauna que allí se alberga es desconocida (Coddington \& Levi, 1991). Las arañas de vegetación son ubicuas y fáciles de colectar, destacando aquéllas que pertenecen a las familias constructoras de telas, debido a que presentan una condición de semi-sesilidad (Oliver \& Beattie, 1993; Wise, 1993). En este grupo encontramos familias como Anapidae, Araneidae, Deinopidae, Linyphiidae, Mysmenidae, Pholcidae, Symphytognathidae, Theridiidae, Theridiosomatidae, Tetragnathidae y Uloboridae (Dippenaar-Schoeman \& Jocque, 1997; Uetz et al., 1999). Asimismo, se caracterizan por una alta diversidad taxonómica dentro de un hábitat mostrando respuestas específicas ante los cambios ambientales (Coddington et al., 1996). Por ello, conocer de qué manera la composición paisajística regional y sus estructuras locales afectan la distribución y los niveles de diversidad de las arañas, puede revelar rasgos importantes del paisaje e identificar aspectos biogeográficos de los ecosistemas (PinkusRendón et al., 2006).

El grado de conocimiento de las arañas en Sudamérica desde el punto de vista faunístico es aceptable y ha revelado niveles altos de diversidad (número de especies y de familias) en áreas subtropicales de Argentina y sur de Brasil, como también de sectores de Buenos Aires y Uruguay (Pérez-Miles et al., 1999; Rodrigues, 2005; Nogueira et al., 2006; Avalos et al., 2007, 2009, 2013; Bonaldo et al., 2007; Podgaiski et al., 2007; Rubio et al., 2008; Rubio \& Moreno, 2010; Grismado et al., 2011; Baldissera et al., 2012; Rubio, 2014). Los estudios sobre arañas exclusivamente en Argentina se enfocaron, en su mayoría, en aspectos taxonómicos y sistemáticos de algunas familias; siendo los trabajos de diversidad principalmente orientados en agroecosistemas con distintos manejos (Liljesthröm et al., 2002; Beltramo et al., 2006; Armendano \& González, 2010; Avalos et al., 2013), muy pocos en áreas naturales protegidas (Rubio et al., 2008; Rubio \& Moreno, 2010) o en ambientes degradados (Avalos et al., 2007). Aún hay mucho por conocer sobre la fauna nativa de arañas en Argentina, y en especial en áreas con un valor agregado (como las yungas) debido a la diversidad ambiental y biológica y al incremento de la pérdida de muchos de sus hábitats.

Sobre la vertiente oriental de las cadenas montañosas de los Andes se extiende el sistema de los bosques nublados y selvas de montaña, llamados globalmente como bosques andinos yungueños (Brown et al., 2006). El límite sur de la distribución de estos bosques se encuentra en el noroeste de la Argentina y el sur de Bolivia, conocidos localmente como selva tucumanoboliviana, selva tucumano-oranense, o simplemente yungas (Brown et al., 2006); para otros autores también conocidas como yungas surandinas (Olson et al., 2001). Las yungas argentinas cuentan con un alto valor de biodiversidad, en el país sólo comparable con la selva misionera, y un elevado grado de endemismo en general (Bertonatti \& Corcuera, 2000). Esta ecorregión se extiende por más de $4000 \mathrm{~km}$ sobre las vertientes orientales de cadenas andinas y subandinas y se muestra en su extremo austral (en el noroeste argentino-NOA) fragmentada y discontinua (Fig. 1). Y a diferencia de lo que ocurre más al norte, donde limita hacia el E con ecorregiones húmedas, en el NOA discurre a modo de corredor entre formaciones xéricas (Chaco Serrano y Puna-prepuna) (Acosta, 2002). En estas selvas de montaña la vegetación predominante varía de acuerdo al gradiente altitudinal y genera la coexistencia de especies de diferentes orígenes biogeográficos a lo largo del gradiente. De este modo, la vegetación de las yungas se organiza en pisos o franjas de características fisonómico-florísticas bien diferenciables: selva pedemontana, selva montana y bosque montano (Brown et al., 2002). Estructuralmente, la selva montana posee estratos más densos y con mayor organización física de la vegetación (lianas, epífitas, musgos, sotobosque más denso, estrato arbóreo, etc.) (LIEY \& FVSA, 2000). En la provincia de Salta el chaco muestra una ingresión en el Valle de Lerma, provocando la fragmentación de las yungas en parches y creando una compleja interfaz, con marcados contrastes en cortas distancias. Algunos autores sostienen que en la porción central de la provincia, existe un área de conectividad y de representatividad de las yungas, la cual es área prioritaria para conservar (Brown \& Malizia, 2004). 


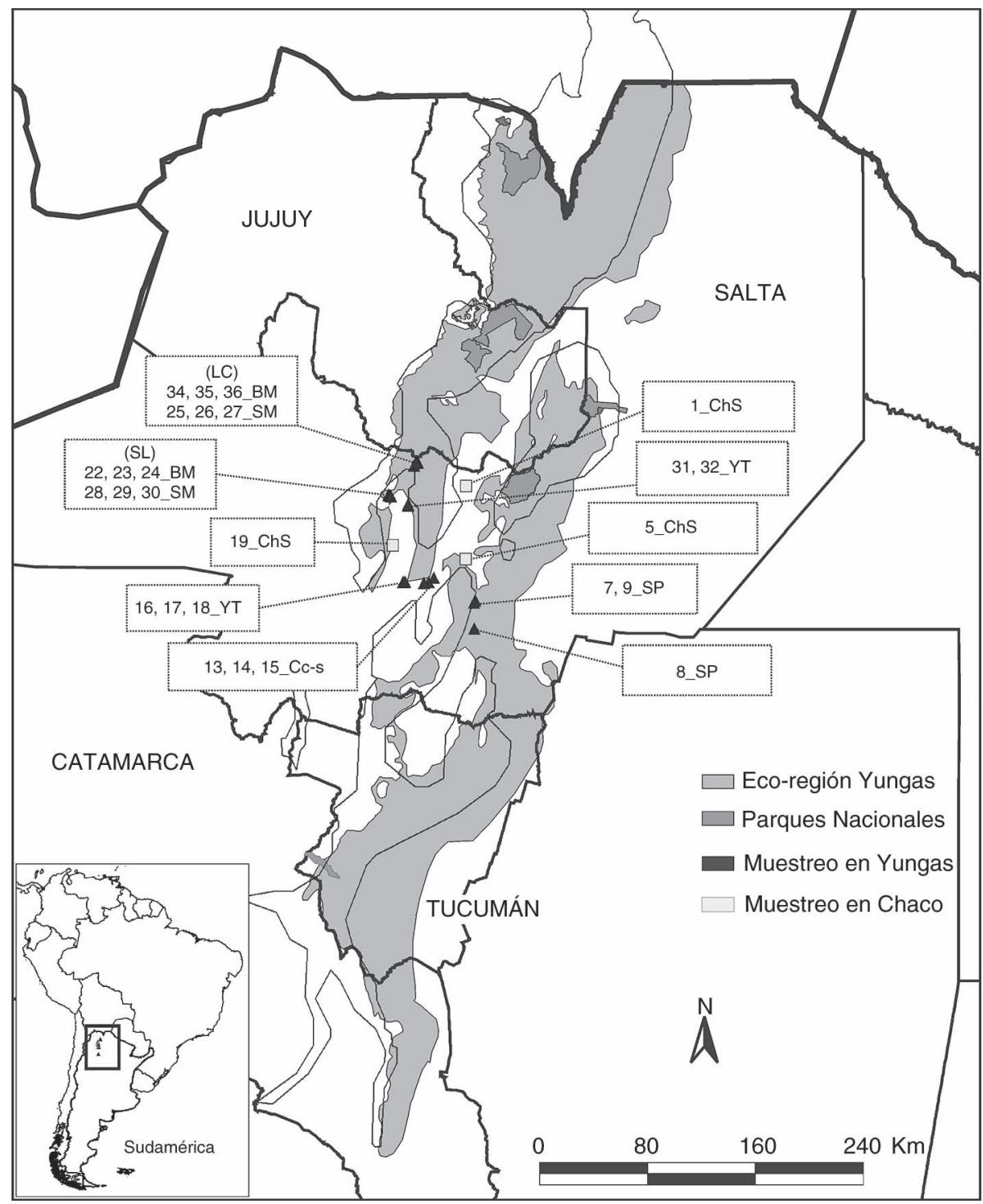

Fig. 1.- Área de estudio y sitios de muestreo (triángulos=Yungas; cuadrados=Chaco Serrano). El área sombreada es una representación de la Eco-región de las Yungas en el noroeste de Argentina, según Brown et al. (2006), y el contorno delgado negro, según Olson et al. (2001). Cada número constituye un sitio al cual se hace referencia en el texto y otras figuras. LC=localidad La Caldera; SL=localidad San Lorenzo.

Fig. 1.- Study area and sampling sites (triangles=Yungas; squares=Chaco Serrano). The shaded area is a representation of the Eco-region of Yungas in northwestern Argentina according to Brown et al. (2006), and the thin black outline according to Olson et al. (2001). Each number represents a site which is referred to in the text and other figures. $L C=L a$ Caldera locality; $S L=S a n$ Lorenzo locality.

Hasta el presente, la unidad biogeográfica de las Yungas ha sido caracterizada mayormente por sus componentes florísticos; que permiten identificarla como una unidad definida (Cabrera \& Willink, 1973). La presencia de otros grupos taxonómicos para caracterizar a las yungas como una región natural, ha sido documentada mediante algunas menciones implícitas y esporádicas de especies de artrópodos (Cuezzo et al., 2007). Por su parte, otros autores defienden la entidad propia de las yungas en base a su composición y diversidad faunística: Acosta (2002) obtiene que los opiliones de las yungas conforman un grupo muy bien caracterizado y de alta diversidad; Morrone (2006) compila una lista de taxones aparentemente endémicos que incluye cuatro especies de insectos; Navarro et al. (2009) reconocen 29 especies de insectos propios de las yungas a partir de un método cuantitativo de identificación de áreas endémicas. De este modo, en la yungas argentinas diversos grupos de artrópodos, excluyendo las arañas, han recibido atención en las últimas décadas, pero indudablemente muchas especies restan de ser encontradas y nuevos taxa descubiertos. Estas selvas de montaña están mayoritariamente aisladas de ecosistemas similares por terrenos empinados y tierras bajas intermedias con contrastes ambientales; son áreas muy importantes por su gran riqueza y altos niveles de endemismo 
(Bertonatti \& Corcuera, 2000; Brown et al., 2006; Cuezzo et al., 2007).

La medición y análisis de la distribución de la biodiversidad implica una tarea difícil, ya que hay varias causas (ecológicas, históricas, etc.) que pueden explicar diferentes aspectos de la diversidad (riqueza de especies, dominancia, rareza) a diferentes escalas espaciales (parches, hábitat, paisajes, ecosistemas, regiones biogeográficas), en diferentes extensiones geográficas (local, regional, global) y en diferentes grupos de organismos (especies, comunidades, taxones de alto rango) (Humphries et al., 1995). El enfoque de este trabajo se define por la ecología de comunidades de arañas, a partir de datos cuali-cuantitativos derivados de muestreos sistematizados en el área central de las yungas salteñas. De esta manera, se intenta reunir conocimiento sobre las arañas de vegetación de yungas con aplicabilidad en estudios ecológicos acerca de las relaciones funcionales entre especies y ambientes (Guisan \& Zimmermann, 2000; Austin, 2002).

Los objetivos de esta contribución son: i) describir la estructura básica de las comunidades de arañas de vegetación en los diferentes ambientes estudiados, en términos de abundancia y riqueza de especies, y comparando índices de diversidad $\alpha$, de reemplazo y otras medidas de $\beta$-diversidad; ii) identificar si los cambios de ambientes de la selva de yungas afectan la diversidad y agrupamiento de arañas del follaje; y iii) analizar variaciones temporales de las comunidades de arañas a lo largo de un año y posibles afinidades de las especies y familias por los diferentes ambientes de las selvas de yungas. Cómo hipótesis se proponen: a) las especies de arañas no se distribuyen de manera homogénea a lo largo de los diferentes ambientes/sectores de las selvas de yungas, mostrando grados de afinidad con algunos de ellos; y b) las estructuras de las comunidades de arañas en los distintos sitios y/o regiones de yungas son diferentes, pero sitios próximos y/o similares en estructura de la vegetación muestran una diversidad y agrupamiento de arañas semejantes.

\section{Material y métodos}

ÁREA DE ESTUdio: Tradicionalmente las áreas que han sido ubicadas dentro de las selvas de montañas o yungas en Argentina son denominadas como "yungas sensu stricto", se organizan en franjas o pisos de vegetación según sus características fisonómicas florísticas: selva pedemontana, selva montana y bosque montano (Brown et al., 2002, 2006). Dichas yungas sensu stricto se caracterizan por un fuerte gradiente altitudinal que tiene por correspondencia un importante gradiente climático, topográfico, edáfico y en la composición específica de la vegetación (Brown et al., 2006). A estas selvas deben sumarse las yungas en transición, relativamente más secas, generalmente más simples en estructura y menos diversas, que comúnmente aparecen formando ecotonos con ambientes del chaco serrano (Brown et al., 2006). Otra de las áreas transicionales o marginales de las yungas, más secas, con aptitud agrícola elevada, es el área de conectividad entre los sectores centro y sur de las yungas; este sistema es una de las áreas ecotonales que permiten la conectividad entre los distintos sectores de selvas húmedas (Brown et al., 2002).

Las descripciones generales de cada uno de los estratos/ambientes que representan el área de estudio se detallan a continuación. 1) Selva pedemontana (SP): Ocupa los sectores entre los 400 y $700 \mathrm{~m}$ de elevación en el piedemonte y las serranías de escasa altitud. La flora se halla empobrecida por la presión antrópica (Chebez, 2005). 2) Selva montana (SM): Ocupa las laderas de las montañas entre los 700 y los $1500 \mathrm{~m}$ de elevación y representa la franja altitudinal de máximas precipitaciones pluviales (más de $2000 \mathrm{~mm}$ anuales). En general, es un bosque con predominio de especies perennifolias y con una estacionalidad hídrica menos marcada que la selva pedemontana. La flora es exuberante, destacándose los helechos, musgos, líquenes y epífitas. 3) Bosque montano (BM): Representa el piso ecológico de los "bosques nublados" propiamente dichos (entre los 1500 y 3000 m de elevación) y muestra el paisaje con mayor heterogeneidad estructural dada por distintos estadíos sucesionales (Chebez, 2005). Las especies vegetales comunes son de distribución andina, de origen austral (gondwánico) y boreal (holártico). 4) Yungas en transición (YT): En las yungas argentinas se observa un ecotono entre la selva pedemontana y la vegetación chaqueña, la cual está sufriendo una paulatina invasión de elementos de yungas. Además de las especies típicas de la vegetación chaqueña, en este ambiente se pueden encontrar especies características de las áreas bajas de las yungas. 5) Área de conectividad centro-sur (Cc-s): Corresponde a una de las áreas que por su ubicación estratégica son de potencial interés para la conservación de las yungas (Brown et al., 2002). Esta área comprende la cuenca de los ríos Juramento y Piedras, con sectores de bosque chaqueño serrano, y actúa como conector entre los sectores central y sur de las yungas en sentido estricto (Cc-s en Fig. 1). 6) Chaco Serrano (ChS): En general el bosque serrano está dominado por gran cantidad de cactáceas y leguminosas espinosas especialmente en el norte (Brown et al., 2006). En suelos modificados abundan los pastos, que alcanzan más de tres metros de altura (Chebez, 2005). El fuego, natural o provocado, y los cultivos son importantes factores que actúan como modeladores del paisaje. En muchos casos se eliminan áreas boscosas con fines agrícolas.

Toma DE MUESTRAs: Se realizaron muestreos sistematizados (cuali-cuantitativos), en la porción central de la provincia de Salta (Fig. 1), que abarcan unos $6300 \mathrm{~km}^{2}$ donde están representados los diferentes ambientes de las yungas (triángulos en Fig. 1). Asimismo, y con fines comparativos, se incorporó 
el ChS dentro del universo de estudio; se dispuso de muestreos sistematizados en diferentes sitios de esta ecorregión, los cuales fueron usados como valores de contraste en distintos análisis y resultados (cuadrados en Fig. 1). Para unificar terminología, los distintos estratos o pisos altitudinales (SP, SM, $\mathrm{BM}, \mathrm{YT}$ ) y otros ambientes muestreados (Cc-s, ChS) son considerados con el término de "ambientes". En cada localidad se seleccionaron sitios que estuvieran separados entre sí por no menos de $1 \mathrm{~km}$. Así fueron muestreados 26 sitios, 3 de $\mathrm{ChS}$ y 23 de yungas que representan diferentes situaciones orográficas y condiciones ambientales de la ecorregión dentro del área en estudio. En cada uno de los sitios seleccionados se realizaron muestreos estacionales durante un año (abril 2006-marzo 2007) de la siguiente manera: 10 muestras con G-Vac (garden-vaccum) sobre vegetación, con el propósito de lograr la mayor representatividad de los grupos de arañas desde el suelo hasta una altura de $2.5 \mathrm{~m}$, aproximadamente. Las muestras de G-Vac fueron tomadas con un aspirador Sthil ${ }^{\mathbb{}}$ con tubo de $1.10 \mathrm{~m}$ de longitud y $12 \mathrm{~cm}$ de diámetro (caudal $710 \mathrm{~m}^{3} / \mathrm{h}$ ), y cada muestra fue la succión de la vegetación en un área de un metro cuadrado por el término de un minuto. De esta manera 1040 muestras representaron la totalidad del muestreo. El material colectado fue colocado en bolsas de polietileno con alcohol etílico al 70\%, debidamente rotulado, y las muestras fueron consideradas como independientes y trasladadas al laboratorio para su procesado (limpieza, separación de arañas de otros artrópodos y acondicionamiento y fijación en alcohol $80 \%$ ). Los especímenes testigo ("voucher") están depositados en la Colección de Arácnidos del Instituto de Biología Subtropical (IBSI-Ara, G. Rubio).

BASE DE DATOS: Del material araneológico recolectado se consideraron tanto adultos como juveniles. En este último caso su inclusión y asignación a nivel genérico o morfo-específico fue dependiente del grado de madurez de los especímenes y de la capacidad de resolución por comparación morfológica directa con los adultos (ver consideraciones más adelante); su inclusión fue considerada siguiendo los criterios de Jiménez-Valverde \& Lobo (2007) para obtener estimaciones reales de diversidad y riqueza de especies. De esta manera, el material fue discriminado en una primera instancia en especies (="morfoespecies" cuando no fueron posibles de determinar) utilizando una base de datos de fotografías digitalizadas con los caracteres distintivos, generada con el programa TAXIS 3.5 (Meyke, 1999-2004). A lo largo del trabajo se hace referencia a cada morfoespecie con la abreviación "msp" seguida de un número (no necesariamente correlativo) que corresponde al ingreso en la base de datos, ejemplo: Any msp36 en los casos que sólo se identificó la familia (colocando las tres primeras letras correspondientes, Any $\rightarrow$ Anyphaenidae), y Sanogasta msp190 cuando se identificó a nivel genérico.
CONSIDERACIONES SOBRE EL USO DE JUVENILES: Si bien la primera dificultad al trabajar con arañas radica en el dimorfismo sexual, el punto más crítico está en la inclusión de los juveniles en los análisis, lo cual ha traído algunos debates en congresos (Labarque et al., 2010; Ramírez et al., 2010). El uso de juveniles reduce la pérdida de información en muchos análisis de diversidad (Jiménez-Valverde \& Lobo, 2007) y, de acuerdo a un criterio personal basado en la experiencia del autor, se va corrigiendo y ganando precisión conforme avanza el procesado de muestras y separación de arañas. Asimismo, en un monitoreo anual, existe un gradiente de madurez en las arañas juveniles que permite una selección objetiva de especie-ejemplares a partir del cotejo entre juveniles y con los adultos según caracteres morfológicos, patrones de coloración y frecuencias cualitativas compartidas. Esto es ecuánime siempre que sea realizado por personas con conocimientos y experiencia dentro del rango taxonómico (e.g. Orden Araneae) del grupo en estudio. El principal inconveniente que radica en el uso de juveniles es que el procesado de material requiere el triple de tiempo hasta que se ajusta la base de datos.

ANÁlISIS DE DATOS: La riqueza de especies es una medida natural y simple para describir la comunidad y la diversidad regional (Magurran, 2004). Se generaron curvas de rarefacción, con error estándar, basadas en el número de individuos con el programa EstimateS 8 (Colwell, 2006) para comparar las diversidades entre los distintos ambientes. Con el mismo programa se calculó la riqueza potencial de especies por medio de ocho estimadores no paramétricos (nómina en Tabla 1 ) en cada uno de los ambientes. Estos valores fueron considerados en términos de promedio $(\mathrm{X})$ y mediana $\left(\mathrm{M}_{\mathrm{e}}\right)$ y fueron comparados para analizar cuán completo (completitud) es el inventario realizado, ya que en todo inventario hay especies no registradas (Chao et al., 2000). Para describir la estructura de las comunidades en términos de abundancia proporcional de cada especie se obtuvieron modelos matemáticos (diagrama rango-abundancia) con el programa Species Diversity \& Richness 3.02. El ajuste de los datos empíricos a la distribución subyacente a cada modelo se obtuvo mediante pruebas de bondad de ajuste $\mathrm{x}^{2}$ (Magurran, 2004). Los distintos modelos difieren en cuanto a las interpretaciones biológicas y estadísticas que asumen de los datos (Krebs, 1989; Moreno, 2001; Magurran, 2004). Se calcularon índices de diversidad, riqueza, equitabilidad y dominancia usando los programas Past 1.18 (Hammer et al., 2003) y BioDiversity Pro 2.0 (McAleece, 1999) para cada comunidad comparando entre ambientes; los intervalos de confianza fueron obtenidos por procedimientos de "bootstraping" permitiendo comparaciones estadísticas. Se usó el índice de complementariedad (Colwell \& Coddington, 1994) y los coeficientes e índices de similitud Jaccard, Sorensen, Morisita y Bray-Curtis (Magurran, 2004) para comparar la composición taxonómica de las comunidades de 
arañas. El grado de asociación o similitud entre sitios, ambientes y comunidades se analizó en base a los individuos utilizando las técnicas ecológicas de multivariados de ordenamiento Escalado Multidimencional no Métrico (NMDS) y Análisis de Correspondencia Detendenciado (DCA), y de clasificación con Análisis de Clúster en base a los índices de similitud mencionados anteriormente (Jongman et al., 1995). Estos análisis fueron construidos con el programa Past 1.18.

\section{Resultados}

INVENTARIO DE ESPECIES Y COMPOSICIÓN: Se obtuvieron 6412 arañas en las yungas, que representaron 188 especies de 34 familias (Tabla 1). Theridiidae $(n=1087)$, Anyphaenidae $(\mathrm{n}=812)$, Linyphiidae $(\mathrm{n}=757)$, Tetragnathidae $(\mathrm{n}=723)$ y Araneidae $(\mathrm{n}=536)$ fueron dominantes y representaron el 61.06\% (3915/6412) de la abundancia total en las yungas. Las familias con mayor riqueza fueron Araneidae $(S=34)$, Theridiidae $(S=31)$, Salticidae $(\mathrm{S}=21)$, Anyphaenidae $(\mathrm{S}=14)$ y Thomisidae $(\mathrm{S}=12)$ que representaron el $59.57 \%(112 / 188)$ de la riqueza total. Las especies dominantes fueron Chibchea salta Huber, 2000 (Pholcidae [abundancia relativa 8.09\%]), Dubiaranea msp111 (Linyphiidae [6.56\%]), Mysmena msp110 (Mysmenidae [6.39\%]), cf. Temnidia msp131 (Anyphaenidae [5.69\%]), cf. Misumenops msp27 (Thomisidae [4.54\%]), Leucauge cf. venusta (Tetragnathidae [4.47\%]), cf. Chrisometa msp187 (Tetragnathidae [4.46\%]) y Dubiaranea difficilis (Mello-Leitão, 1944) (Linyphiidae [4.07\%]).

En el ChS se colectó un total de 1162 arañas que representan 79 especies y 18 familias. Anyphaenidae, Araneidae, Philodromidae, Thomisidae y Theridiidae fueron dominantes; mientras que las familias con mayor riqueza fueron Araneidae, Anyphaenidae, Salticidae, Theridiidae y Oxyopidae. Se obtuvieron 15 especies exclusivas de este ambiente. Las especies dominantes fueron cf. Temnidia msp131, Paracleocnemis msp75 (Philodromidae), Metazygia viriosa (Keyserling, 1892) (Araneidae), cf. Misumenops msp27, Parawixia bistriata (Rengger, 1836) (Araneidae), Oxyopes msp11 (Oxyopidae), Araneus msp69 y Ocrepeira msp199 (Araneidae). La nómina completa de especies/morfoespecies se muestra en el Apéndice 1.

Tabla 1.- Valores de diversidad $\alpha$ de las comunidades de arañas en los distintos ambientes de las yungas de Salta (Argentina). La última columna muestra los valores para el ChS a modo de contraste. $\mathbf{X}=$ según el promedio; $\mathbf{M}_{\mathrm{e}}=$ según la mediana; "singletons" y "doubletons"=especies representadas por uno y dos individuos.

Table 1.- Values of $\alpha$ diversity of spider communities in the different environments of the Yungas of Salta (Argentina). The last column shows the ChS values for a contrast. $\mathbf{X}=$ according to the average; $\mathbf{M}_{\mathbf{e}}=$ according to the median; singletons and doubletons=species represented by one and two individuals.

\begin{tabular}{|c|c|c|c|c|c|c|c|}
\hline Medidas de diversidad $\alpha$ & Cc-s & YT & SP & SM & BM & $\begin{array}{l}\text { totales } \\
\text { Yungas }\end{array}$ & ChS \\
\hline Riqueza observada & 70 & 108 & 98 & 81 & 86 & 188 & 79 \\
\hline Riqueza estimada $(X)$ & 97.17 & 134.00 & 139.54 & 93.27 & 119.77 & 207.87 & 108.76 \\
\hline Riqueza estimada $\left(\mathrm{M}_{\mathrm{e}}\right)$ & 96.82 & 132.49 & 139.46 & 91.83 & 114.47 & 205.98 & 109.25 \\
\hline ACE (abundance-based coverage estimator) & 95.38 & 131.95 & 139.38 & 91.78 & 110.61 & 204.08 & 117.06 \\
\hline ICE (incidence-based coverage estimator) & 102.33 & 139.98 & 153.74 & 96.18 & 114.06 & 207.71 & 123.03 \\
\hline Chao 1 & 85.79 & 126.9 & 145.12 & 90.55 & 140.17 & 204.25 & 104.2 \\
\hline Chao 2 & 98.76 & 133.02 & 152.32 & 91.88 & 153.38 & 207.75 & 109.74 \\
\hline Jackknife $1^{\circ}$ orden & 99.75 & 140.84 & 136.68 & 98.93 & 114.88 & 217.97 & 109.74 \\
\hline Jackknife $2^{\circ}$ orden & 115.6 & 153.8 & 161.37 & 103.94 & 138.7 & 226.97 & 126.57 \\
\hline Bootstrap & 83.3 & 123.52 & 114.8 & 89.82 & 97.99 & 203.22 & 92.6 \\
\hline Michaelis-Menten (media) & 96.46 & 121.95 & 112.92 & 83.05 & 88.34 & 190.97 & 87.15 \\
\hline completitud inventario (\%) $\mathrm{M}_{\mathrm{e}}$ & 72.30 & 81.52 & 70.27 & 88.21 & 75.13 & 91.27 & 72.31 \\
\hline completitud inventario (\%) X & 72.04 & 80.60 & 70.23 & 86.85 & 71.81 & 90.44 & 72.64 \\
\hline índice de Shannon $\left(\mathrm{H}^{\prime}\right)$ & 3.3 & 3.64 & 3.65 & 3.33 & 3.19 & 4.01 & 2.93 \\
\hline Equitabilidad & 0.78 & 0.78 & 0.80 & 0.76 & 0.72 & 0.77 & 0.6711 \\
\hline Dominancia Berger-Parker & 0.19 & 0.12 & 0.12 & 0.13 & 0.14 & 0.08 & 0.2866 \\
\hline índice de riqueza Margalef & 11.52 & 14.92 & 14.3 & 10.5 & 11.35 & 21.33 & 11.05 \\
\hline \multicolumn{8}{|l|}{ Otros valores } \\
\hline $\mathrm{n}^{\circ}$ de muestras & 120 & 200 & 120 & 240 & 240 & 920 & 120 \\
\hline $\mathrm{n}^{\circ}$ de individuos & 399 & 1301 & 881 & 2041 & 1790 & 6412 & 1162 \\
\hline $\mathrm{n}^{\circ}$ de "singletons" & 25 & 28 & 35 & 15 & 26 & 26 & 28 \\
\hline $\mathrm{n}^{\circ}$ de "doubletons" & 18 & 19 & 13 & 10 & 5 & 19 & 14 \\
\hline
\end{tabular}


En yungas el esfuerzo de muestreo representó una proporción individuos:especies de 34.11:1. Por ambiente, en SP fue de 8.99:1, en SM 25.2:1, en BM 20.81:1, en Cc-s 5.7:1 y en YT 12.05:1. En el $\mathrm{ChS}$ la proporción fue de 14.71:1. De la estimación de riqueza y completitud de inventarios (proporción de riqueza esperada representada por la riqueza observada), surgieron los siguientes valores (Tabla 1; Fig. 2): en Cc-s el rango de riqueza estimada (Sest) fue de 83.3-102.3 especies con una completitud (en base a la riqueza estimada promedio) del $72.04 \%$ en la riqueza observada (Sobs), en YT se estimó $121.9-153.8$ especies (80.6\%), en SP 112.9-161.4 (70.23\%), en SM 83-103.9 (86.85\%) y en BM 88.3-153.4 (71.81\%). Por su parte, en el ChS se estimó un rango de 87.1-126.6 especies, con una completitud del inventario de $72.64 \%$. El inventario más completo correspondió a la SM, mientras que en $\mathrm{Cc}-\mathrm{s}$, SP y BM se estimaron las comunidades de arañas que albergarían potencialmente más especies respecto de la riqueza observada. Para la totalidad de las yungas, la proporción de especies observadas:estimadas (X de especies estimadas) sugiere que se esperarían de ser colectadas al menos un $10 \%$ de especies más en el área de muestreo. La cantidad más alta de "singletons" (especies representadas por un solo individuo) se registró en SP $(\mathrm{n}=35)$ y de "doubletons" (especies representadas por dos individuos) en YT y Cc-s (19 y 18 respectivamente). La completitud para toda el área estudiada de yungas fue superior al $90 \%$, siendo en el ChS más baja y con alto número de singletons y doubletons (Tabla 1).

DiVERSIDAD $\alpha$ Y ESTRUCTURA DE LAS COMUNIDADES: La distribución de las comunidades de arañas se ajusta a los modelos log-normal y serie logarítmica. Los ajustes son estadísticamente significativos $\left(\mathrm{x}^{2}=2.36-5.01\right.$, $\mathrm{p}>0.05$ ), con excepción en el ChS para la serie logarítmica $\left(\mathrm{x}^{2}=12.96, \mathrm{p}=0.047\right)$ en cuya comunidad aumentan los niveles de dominancia (Tabla 1).

En términos de riqueza específica, las curvas de rarefacción mostraron diferencias entre comunidades (Fig. 3). Del esfuerzo de muestreo estandarizado al $1^{\circ}$ nivel de corte (incluyendo la comunidad menos abundante; $n=399$ ) se observó que YT, SP y $\mathrm{Cc}-\mathrm{s}(\mathrm{S}=72.7,72.5,70$ respectivamente) fueron consistentemente más ricas que $\mathrm{BM}, \mathrm{SM}$ y $\mathrm{ChS}$ $(\mathrm{S}=52.6,52.5,51.4)$. Estas diferencias se mantuvieron
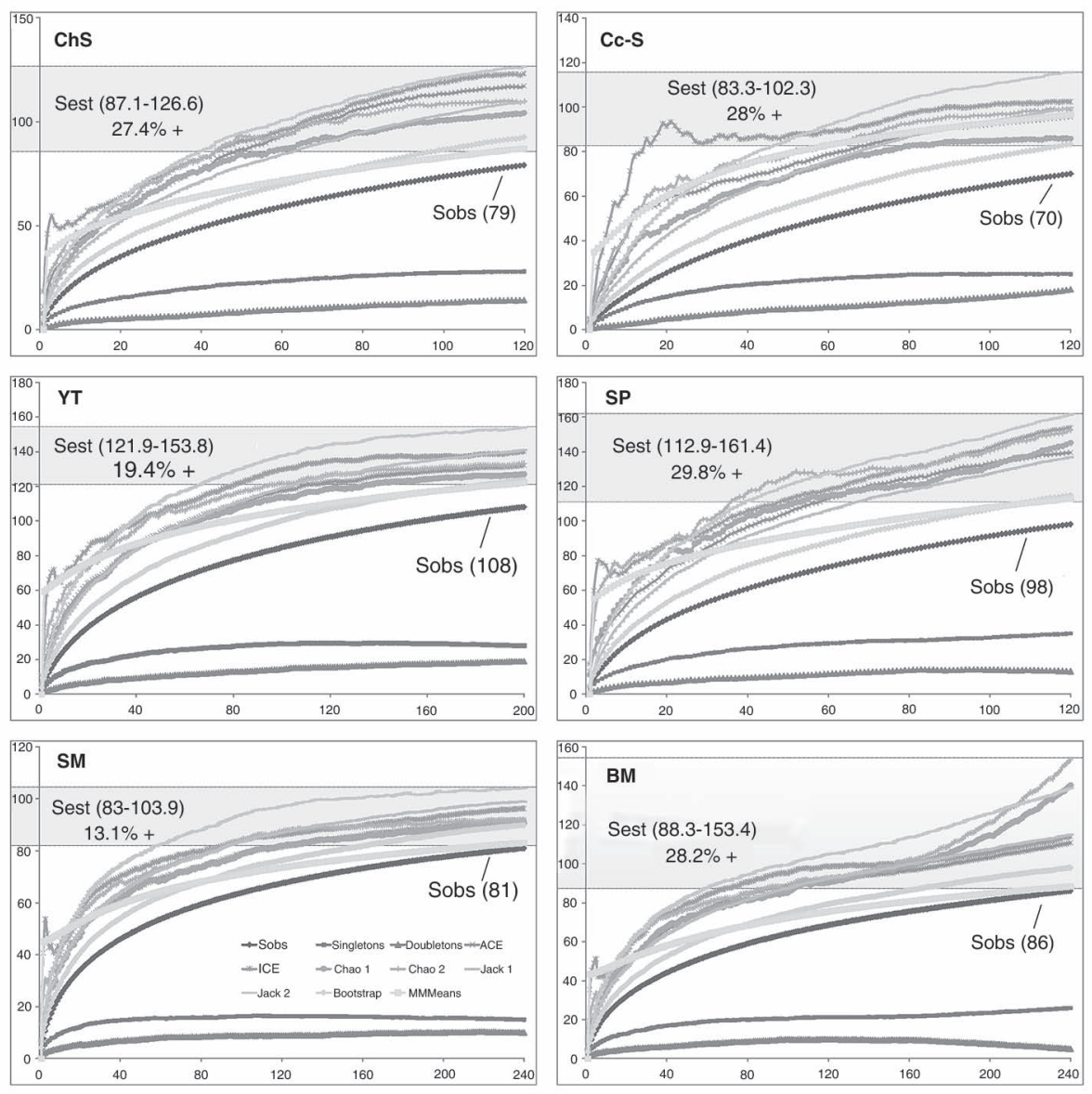

Fig. 2.- Curvas de acumulación de especies (Sobs) y comparación con el rango de riqueza estimada (Sest) para cada ambiente a partir de ocho estimadores no paramétricos en función del esfuerzo acumulativo de muestreo $\left(n^{\circ}\right.$ de muestras en abscisas, $n^{\circ}$ de especies en ordenadas).

Fig. 2.-Species accumulation curves (Sobs) and comparison with the range of estimated richness (Sest) for each environment, from eight nonparametric estimators based on the cumulative sampling effort ( $n^{\circ}$ of samples on abscissa- $x$ axis; $n^{\circ}$ of species on ordinate- $y$ axis). 


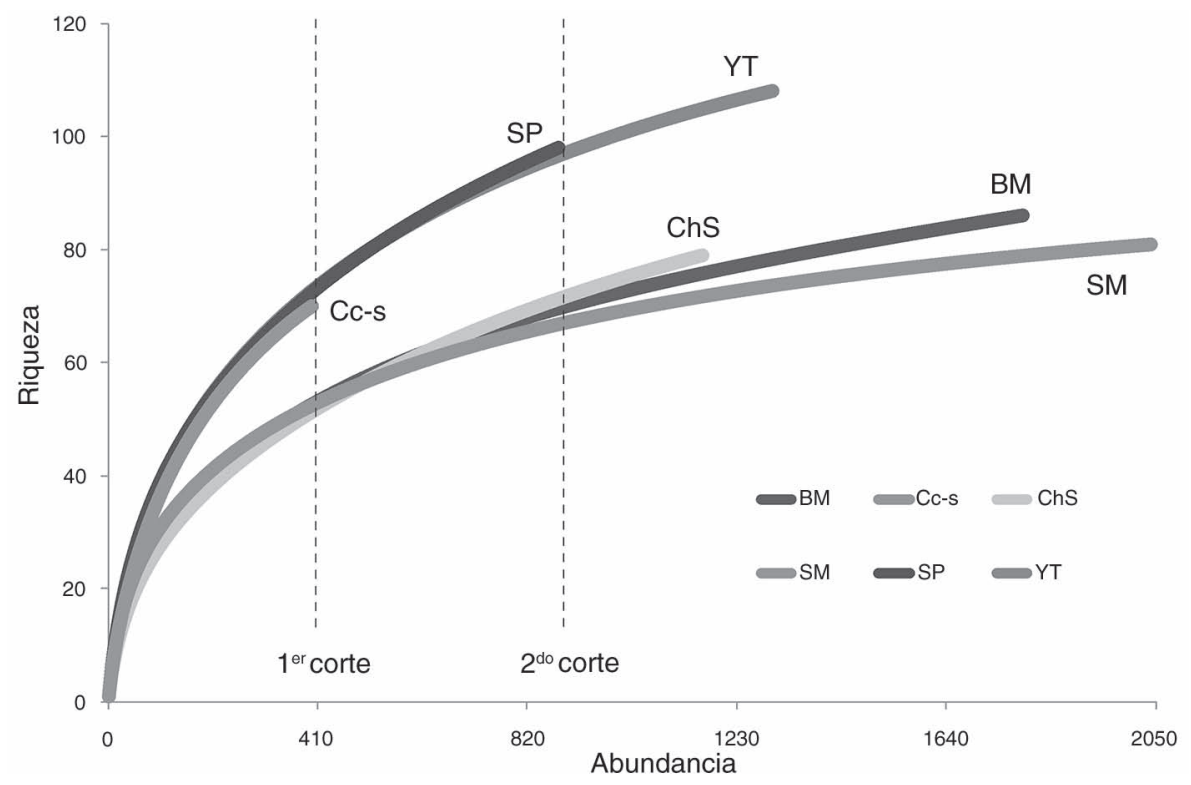

Fig. 3.- Riqueza de especies de arañas en los ambientes de Yungas (Salta, Argentina) a través de las curvas de rarefacción en base al número de individuos. Se incluye el ChS en un marco comparativo.

Fig. 3.- Species richness of spiders in the environments of Yungas (Salta, Argentina) through rarefaction curves based on number of individuals. The ChS is included in a comparative framework.

al excluir la comunidad menos abundante (Cc-s) y dejando en su lugar a SP con una ganancia de información a partir de los 881 individuos (al $2^{\circ}$ nivel de corte): $\mathrm{S}=98$ en SP y 96.5 en YT; $\mathrm{S}=71.3,69.6,66.9$ en ChS, BM y SM. Los solapamientos de las curvas de SP con YT y de ChS, BM y SM entre sí indicaron las diferencias significativas de riqueza consistentes entre estos dos grupos de ambientes (Fig. 3).

Los ambientes SP y YT fueron los más diversos (índices de Shannon y Margalef en Tabla 1; Bootstrap $\mathrm{p}<0.05$ ); en segundo lugar siguieron SM, Cc-s y $\mathrm{BM}$, a su vez significativamente más diversos que el ChS (Bootstrap p<0.05). La mayor equitabilidad se obtuvo en SP significativamente diferente al $\mathrm{ChS}$ y BM (Bootstrap p $<0.05)$. Los ambientes Cc-s y YT también presentaron alta equitabilidad superando consistentemente al ChS (Tabla 1). La mayor dominancia se obtuvo en el ChS (índice de Berger-Parker), mostrando diferencias significativas con la mayoría de los ambientes (Bootstrap $\mathrm{p}<0.05$ ); asimismo, entre los ambientes propios de yungas se observó al $\mathrm{Cc}$-s con un índice de dominancia consistentemente mayor (Bootstrap $\mathrm{p}<0.05)$. Los niveles de diversidad $\left(\mathrm{H}^{\prime}\right)$ no presentaron correlación significativa con las distintas altitudes en que se encontraron los ambientes $(\mathrm{r}=-0.037)$.

VARIACIONES A LO LARGO DEL AÑO: La mayor cantidad de arañas en las yungas se obtuvo en otoño $(n=2030)$, seguido por el verano $(n=1965)$, invierno $(n=1262)$ y primavera $(\mathrm{n}=1155)$. En verano se obtuvo la mayor riqueza $(S=146)$. En otoño y primavera se capturaron 120 y 115 especies, respectivamente, y en invierno se observó la menor riqueza ( $\mathrm{S}=97)$.
La familia Theridiidae fue dominante en otoño y primavera $(22.4 \% ; 16.8 \%)$, seguida por Pholcidae y Anyphaenidae para estas estaciones climáticas (16.1\%; $16.4 \%$ ). Linyphidae fue más abundante en invierno (25.7\%), sin estar entre las ocho familias dominantes del verano, donde Anyphaenidae y Tetragnathidae fueron superiores $(15.8 \% ; 14.3 \%)$. Al considerar las especies con más de 20 individuos, se observaron variaciones de abundancia con relación a la época del año. Chibchea salta, Mysmena msp110, Anelosimus msp118, Mangora cochuna Levi, 2007, Josa msp126, las Theridiidae msp52 y msp161, cf. Maro msp108 y Lobizon ojangureni Piacentini \& Grismado, 2009 mostraron altas abundancias en otoño, duplicándose con respecto a las demás estaciones; no obstante, en el caso de Anelosimus msp118 su abundancia en otoño estuvo asociada a una alta desviación estándar. Las especies de Dubiaranea (D. msp111, D. difficilis) y Leucauge cf. venusta tuvieron más individuos en invierno. Sanogasta msp190, cf. Chrysometa msp187, Paracleocnemis msp75, Tet_msp57 y Ocrepeira msp43 aumentaron su abundancia en verano. Algunas especies de Araneidae mostraron mayor abundancia en primavera (e.g. Ara_msp230, Araneus msp69 y Metazygia viriosa). Sin embargo, las abundancias de algunas especies se mantuvieron constante a lo largo del año, e.g.: cf. Misumenops msp27, Tmarus estyliferus Mello-Leitão, 1929 y Lyssomanes msp18.

VARIACIONES POR AMBIENTES DE YUNGAS: Tetragnathidae fue dominante en BM (19\%), en SM y SP Theridiidae se registró con más individuos $(20.7 \% ; 26.8 \%)$. Thomisidae fue dominante en YT (22.3\%) y Anyphaenidae en YT y $\mathrm{ChS}(24 \% ; 29.6 \%)$. Pocas especies se mantuvieron 

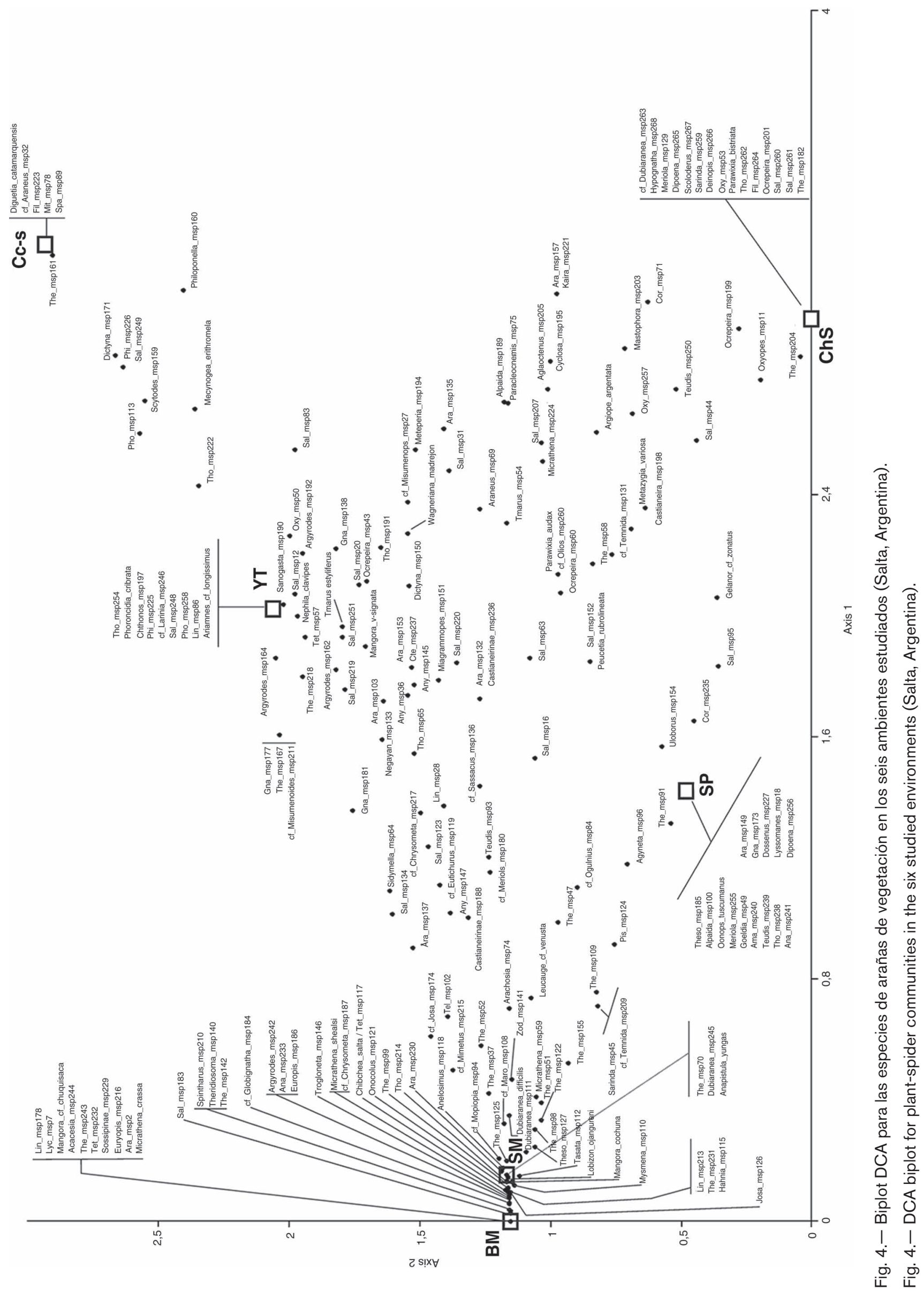
con niveles de abundancia homogéneos para todos los ambientes de yungas: cf. Temnidia msp131, Araneus msp69, Metazygia viriosa y Lencauge cf. venusta. Se observó que las diferencias relevantes en cuanto al número de individuos por especie están más asociadas a grupos de ambientes que a ambientes propios (ver agrupamiento de ambientes-diversidad $\beta$ ). A los pisos de menor altitud de las yungas están asociadas especies de las familias Dictynidae, Oxyopidae, Philodromidae, Uloboridae y Thomisidae (en esta última principalmente del género Tmarus), algunas Anyphaenidae (msp36, msp145 y Sanogasta msp190), Ara_msp135, Mecynogea msp165 y Aglaoctenus msp205.

Linyphiidae, Pholcidae, Mysmenidae, Lycosidae, Hahniidae y Symphytognathidae se asociaron a los ambientes de mayor altitud (SM y BM); a estos ambientes se coligaron las especies Mangora cochuna, Micrathena shealsi Chickering, 1960, Josa msp126, Tasata msp112, ambas especies de Dubiaranea, Lobizon ojangureni, Mysmena msp110, Trogloneta msp146, Chibchea salta, cf. Chrysometa msp187, Spintharus msp210 y Theridiosoma msp140 entre otras. También se observaron otras especies (abundancias $\mathrm{n}>30$ ) asociadas preferentemente a sólo uno de los ambientes: Nephila clavipes (Linnaeus, 1767) en YT (94.1\%), Lyssomanes msp18 en SP (100\%) y The_msp161 en Cc-s (98\%).

Lo antedicho es más completo e integralmente visualizado en las Figs. 4 y 5 (análisis de DCA). La fauna de arañas presentó patrones de asociación hacia los distintos ambientes según su composición específica (Fig. 4) o de rango familiar (Fig. 5); además los análisis reflejaron el agrupamiento de los ambientes según su composición araneo-faunística. En ambos ordenamientos gráficos (Figs. 4 y 5) el primer eje separó claramente los ambientes, y sus taxones asociados, según un gradiente altitudinal inversamente proporcional al valor de dicho eje 1. En la Fig. 4 el eje 1 tuvo un eigenvalor de 0.735 y el eje 2 de 0.227 , explicando ambos ejes el $85.64 \%$ de la variación en los datos; en la Fig. 5, el eje 1 tuvo un eigenvalor de 0.426 y el eje 2 de 0.034 , ambos ejes explican el $94.21 \%$ de la variación.

DIVERSIDAD $\beta$, RECAMBIO y COMPLEMENTARIEDAD: Las comunidades de arañas de BM y SM presentaron la mayor similitud ( $>50 \%$ para los tres índices y coeficientes usados-Tabla 2), dichos ambientes compartieron la mayor cantidad de especies al igual que SP con YT (61 especies c/u), por lo cual sus índices de complementariedad fueron bajos $(0.42 ; 0.58$ respectivamente). Entre los demás ambientes la complementariedad superó el $60 \%$. La menor cantidad de especies compartidas fue observada en BM con ChS, y SM con ChS (21 y 23 especies). Los valores de complementariedad y similitud revelaron en la región una alta diversidad $\beta$ (e.g. complementariedad $\sim 0.69$, considerando que 1 es indicativo de que las comunidades son completamente diferentes).

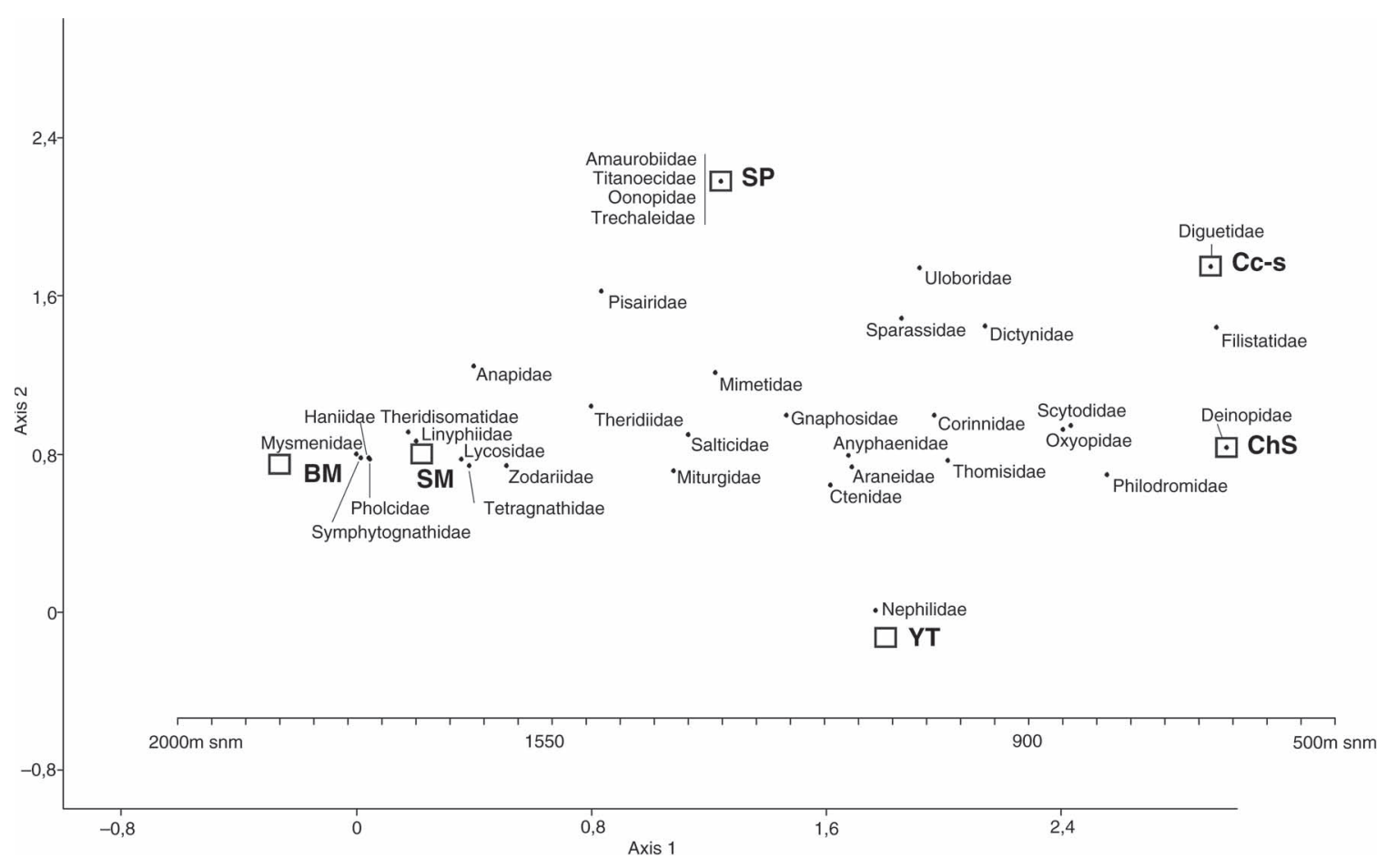

Fig. 5.- Biplot DCA para las familias de arañas de vegetación en los distintos ambientes (Salta, Argentina). La barra de altitud ( $\mathrm{m}$ snm) es aproximativa para ver el gradiente en relación a los ambientes estudiados.

Fig. 5.- DCA biplot for the plant-spider families in the six studied environments (Salta, Argentina). The altitude bar ( $\mathrm{m}$ asl) is approximate to see the gradient in relation to the studied environments. 
Tabla 2.- Valores de diversidad $\beta$, con máximos ${ }^{(+)}$y mínimos ${ }^{(-)}$para las comunidades de arañas de vegetación, comparando entre pares de diferentes ambientes.

Table 2.- Values of $\beta$ diversity, with maximum ${ }^{(+)}$and minimum ${ }^{(-)}$for the plant-spider communities of vegetation, comparing between pairs of different environments.

\begin{tabular}{lccccc}
\hline & $\begin{array}{c}\mathbf{n}^{\circ} \text { de especies } \\
\text { compartidas }\end{array}$ & $\begin{array}{c}\text { coeficiente de } \\
\text { Sorensen (cualitativo) }\end{array}$ & $\begin{array}{c}\text { coeficiente de } \\
\text { Jaccard (cualitativo) }\end{array}$ & $\begin{array}{c}\text { índice de Morisita } \\
\text { (cuantitativo) }\end{array}$ & $\begin{array}{c}\text { índice de } \\
\text { complementariedad }\end{array}$ \\
\hline BM x Cc-s & 27 & 0.35 & 0.21 & $0.06^{(-)}$ & 0.79 \\
BM x SM & $61^{(+)}$ & $0.73^{(+)}$ & $0.58^{(+)}$ & $0.90^{(+)}$ & $0.42^{(-)}$ \\
BM x SP & 43 & 0.47 & 0.30 & 0.30 & 0.70 \\
BM x YT & 41 & 0.42 & 0.27 & 0.13 & 0.73 \\
BM x ChS & $21^{(-)}$ & $0.25^{(-)}$ & $0.15^{(-)}$ & 0.12 & $0.85^{(+)}$ \\
Cc-s x SM & 28 & 0.37 & 0.23 & $0.06(-)$ & 0.77 \\
Cc-s x SP & 36 & 0.43 & 0.27 & 0.42 & 0.73 \\
Cc-s x YT & 51 & 0.57 & 0.40 & 0.71 & 0.60 \\
Cc-s x ChS & 39 & 0.52 & 0.35 & 0.55 & 0.65 \\
SM x SP & 46 & 0.51 & 0.35 & 0.33 & 0.65 \\
SM x YT & 45 & 0.48 & 0.31 & 0.13 & 0.69 \\
SM x ChS & 23 & 0.29 & 0.17 & 0.10 & 0.83 \\
SP x YT & $61^{(+)}$ & 0.59 & 0.42 & 0.62 & 0.58 \\
SP x ChS & 40 & 0.45 & 0.29 & 0.54 & 0.71 \\
YT x ChS & 53 & 0.57 & 0.40 & 0.64 & 0.60 \\
\hline
\end{tabular}

A partir de los 26 sitios de muestreo ( 23 de yungas +3 del $\mathrm{ChS}$ ) se obtuvieron dos grupos principales bien definidos de acuerdo con la similitud cuali-cuantitativa de los agrupamientos de arañas (Fig. 6). El Grupo 1 (similitud Bray-Curtis $=0.45$ ) presenta mayor concentración y está constituido por 12 sitios que corresponden a $\mathrm{SM}+\mathrm{BM}$, sin formar una clara sub-agrupación que separe a estos dos ambientes; no obstante, presenta un débil apartamiento de dos sub-grupos si consideramos las zonas de La Caldera (LC) y San Lorenzo (SL) (Fig. 1). El Grupo 2 (similitud=0.28) tiene menor robustez y mayor dispersión de datos, permitiendo las siguientes sub-agrupaciones (Fig. 6): $\mathrm{G}_{\mathrm{A}}$ (similitud $=0.39$ ) correspondiente a SP, separado de $\mathrm{G}_{\mathrm{B}}(0.29)$ que incluye el subgrupo $G_{B 1}(0.46)$ con los ambientes del ChS, y $\mathrm{G}_{\mathrm{B} 2}(0.35)$ con los ambientes YT y Cc-s, a su vez bien definidos como grupos separados (Fig. 6).

\section{Discusión y conclusiones}

Este es el primer estudio de Argentina que se focaliza en la diversidad de arañas (Araneae) y su distribución en los diferentes estratos de la Ecorregión de las Yungas. La riqueza global observada en este estudio fue de 188 especies y 34 familias en los ambientes que representaron las yungas, lo cual sumado a las especies obtenidas en el Chaco Serrano, resultan en una base de datos conteniendo 203 especies y 35 familias. Si bien las técnicas de colectas de arañas son variadas, los niveles de riqueza observados en las selvas de yungas resultaron equivalentes a los observados en otros estudios en otras áreas subtropicales de Argentina y sur de Brasil, como también áreas de Buenos Aires y Uruguay (Pérez-Miles et al., 1999; Rodrigues, 2005; Nogueira et al., 2006; Avalos et al., 2007, 2009, 2013; Bonaldo et al., 2007; Podgaiski et al., 2007; Rubio et al., 2008; Rubio \& Moreno, 2010; Grismado et al., 2011; Baldissera et al., 2012). Asimismo, la riqueza específica obtenida en esta investigación no difiere a las observadas en otros estudios realizados en selvas tropicales amazónicas (Höfer, 1990; Raizer et al., 2005; Machado Pinto-Leite et al., 2008; Ricetti \& Bonaldo, 2008; Bonaldo \& Dias, 2010; Carvalho \& Avelino, 2010). Aunque en un marco menos comparable, en Argentina existen estudios sobre ecología de comunidades de arañas en agroecosistemas que revelaron niveles de riqueza más bajos, debido sin duda a la pérdida de la heterogeneidad del paisaje: cultivos de soja (Liljesthrom et al., 2002; Beltramo et al., 2006), y alfalfa (Armendano \& González, 2010).

Recientes estudios sobre inventarios de especies de arañas en zonas tropicales son bastante desalentadores en cuanto a lograr una buena completitud aún mediante el uso de técnicas de muestreo complementarias, promediando un $32 \%$ de especies capturadas por un solo individuo (singletons) (Toti et al., 2000; Sørensen et al., 2002; Coddington et al., 2007). Una elevada proporción de singletons significa que una gran cantidad de especies no han sido colectadas todavía (20-50\%, Ramírez, com. pers., 2011). $\mathrm{Si}$ consideramos los ambientes de las yungas en su totalidad, el número de singletons encontrados fue bajo (14\%). No obstante, la situación particular de los ambientes Cc-s, SP, e inclusive el $\mathrm{ChS}$ revelaron 


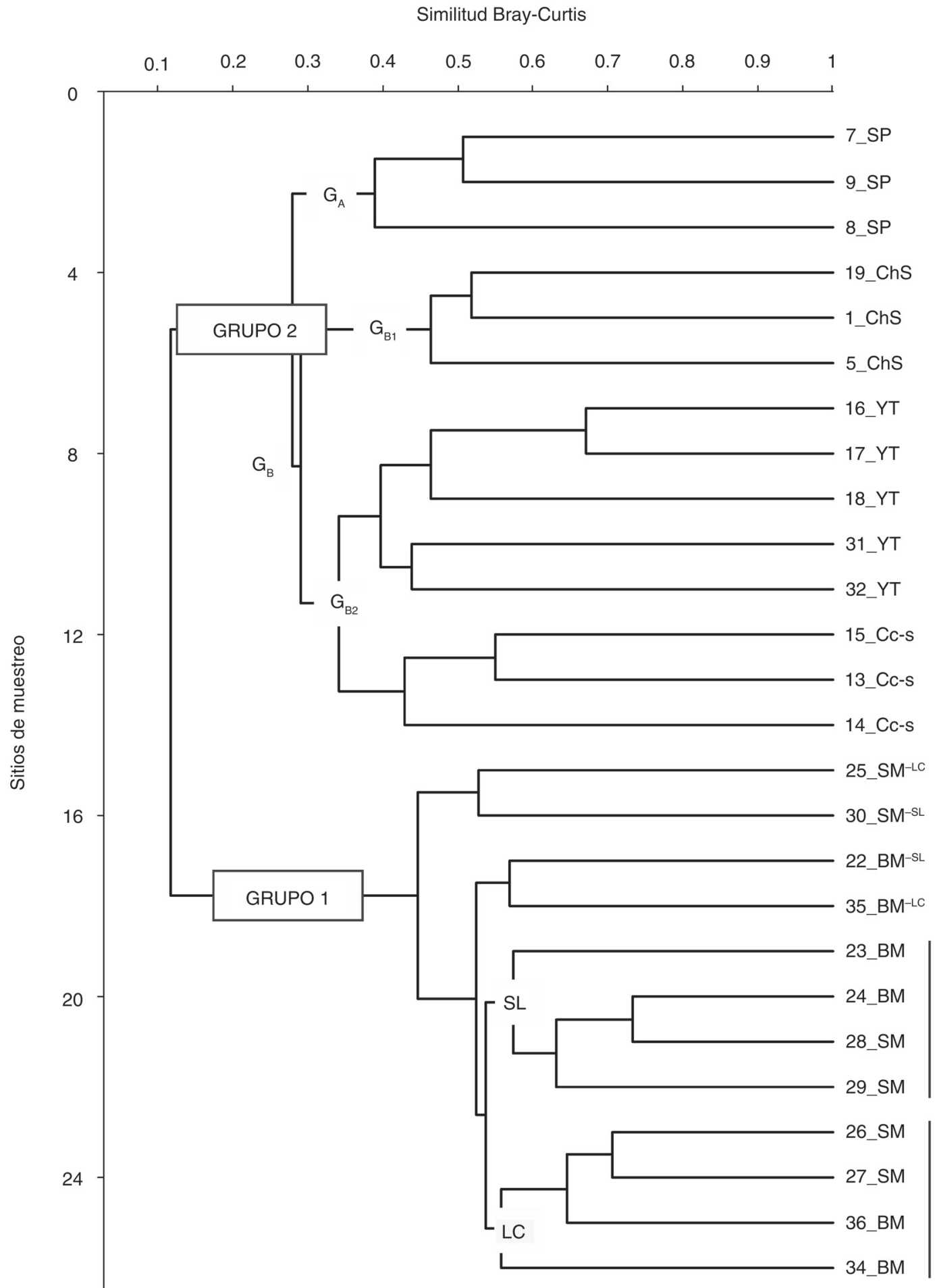

Fig. 6.- Análisis de clasificación (índice de Bray-Curtis) de los sitios $\left(n^{\circ}\right)$ de muestreo en las yungas de Salta (Argentina), incluyendo los ambientes de ChS. $\mathrm{G}_{(\mathrm{A}, \mathrm{B} 1, \mathrm{~B} 2, \mathrm{SL}, \mathrm{LC})}=$ sub-agrupamientos; $\mathrm{SL}=$ localidad San Lorenzo; $\mathrm{LC}=\mathrm{localidad}$ La Caldera.

Fig. 6.- Classification analysis (Bray-Curtis index) of the sampling sites $\left(n^{\circ}\right)$ in the Yungas of Salta (Argentina), including ChS environments. $\mathrm{G}_{(\mathrm{A}, \mathrm{B} 1, \mathrm{~B} 2, \mathrm{SL}, \mathrm{LC})}=$ sub-clusters; $\mathrm{LC}=\mathrm{La}$ Caldera locality; SL=San Lorenzo locality.

valores más altos (35-36\%). Estos niveles de singletons podrían estar indicando una baja completitud, o bien no tener relación con la rareza de especies sino con la detección de especies turistas (Rubio et al.,
2008), y deberse a la fragmentación y distribución en parches propia de las yungas de la provincia de Salta.

Indudablemente el número de singletons tiene ciertos efectos sobre la completitud de los inventarios de 
una comunidad, y los inventarios de arañas aquí obtenidos fueron en general aceptablemente completos. Para los seis ambientes el inventario observado de especies registró más del $70 \%$ de la riqueza esperada, igualando lo obtenido por otros investigadores: 68-89\% (Coddington et al., 1996), 86\% (BlancoVargas et al., 2003), 80\% (Sørensen, 2004), 61-87\% (Rubio et al., 2008), 78-88\% (Cardoso et al., 2008), 71-97\% (Rubio \& Moreno, 2010). Sin embargo, en los ambientes que tuvieron niveles relativamente más bajos de completitud (SP y Cc-s), esto se vio coherente y reflejado según la estimación de la proporción individuos:especies.

La biodiversidad está muy relacionada con la evolución del paisaje, por lo que es importante no desestimar el contexto histórico geográfico de las yungas, región que posee una marcada complejidad y diversidad de ambientes, condicionados en su mayoría por procesos orogénicos, que han formado y modelado el relieve (Brown et al., 2006). Desde el punto de vista de la vegetación, las yungas tienen una estructura compleja a nivel tanto local como regional, y pueden ser caracterizadas a partir de la estructura de las comunidades de arañas. No todos los pisos y áreas tienen los mismos niveles ecológicos de diversidad, equitabilidad y dominancia. Uno podría preguntarse ¿coincide la distribución de las comunidades de arañas con los ambientes tradicionalmente definidos para cada subformación de estas selvas?; puede advertirse, como surgimiento de este estudio, que los ambientes selva y bosque montanos (SM, BM) logran diferenciarse araneológicamente de los ambientes transicionales de las yungas como el área de conectividad centro-sur (Cc-s), las yungas en transición (YT) y la fragmentada selva pedemontana (SP).

Los cambios altitudinales presentan una serie de factores condicionantes para el establecimiento de la biota. Numerosos autores afirman que la diversidad de insectos y arañas disminuye a medida que aumenta la altitud (Janzen et al., 1976; Uetz, 1976; Wolda, 1987; Hartmut et al., 1990; McCoy, 1990). Estas variaciones tienen directa relación con la estructura y diversidad del hábitat que constituyen su complejidad en un ecosistema determinado, encontrando que a mayor número de especies es mayor la complejidad del lugar (Diamond, 1988). El escaso rango de variación altitudinal en que se encontraron los ambientes en este estudio ( $\sim 800-1600 \mathrm{~m}$ snm) mostró exiguas variaciones de la diversidad de arañas en relación al aumento de la altura.

Las arañas del follaje dependen en gran medida de la estructura multi-dimensional de la vegetación, y principalmente las constructoras de telas por la oferta de soportes disponibles (Enders, 1974; Hatley \& Macmahom, 1980; Gunnarsson, 1990). Esta relación permite inferir de forma cualitativa el estado de conservación de algunos ecosistemas y diferenciar las afinidades de las comunidades de arañas (Gunnarsson, 1990).
La selva pedemontana constituye un nexo ecotonal entre el chaco serrano y los pisos de mayor altura (SM y BM), y a pesar de su estructura menos compleja en relación a lo que fue predicho para la SM, resultó ser el piso más diverso y heterogéneo junto con las YT; este ambiente es también considerado por Brown et al. (2006) como el más importante de conservar por su riesgo de desaparición. La presencia elevada de singletons podría también actuar como indicativo de ello. Las observaciones de campo son perfectamente vinculables a los resultados obtenidos en los agrupamientos cualitativos de especies de arañas, e.g. los sitios de selva pedemontana (7, 8 y 9) se diferencian claramente de los de Cc-s y YT en que la vegetación es más húmeda y densa (subgrupo $2 \mathrm{G}_{\mathrm{A}}$ en Fig. 6).

Entre las familias de arañas dominantes en áreas naturales se encuentran generalmente Araneidae, Salticidae, Theridiidae, Anyphaenidae y Thomisidae (Flórez-D., 1998; Podgaiski et al., 2007; Ricetti \& Bonaldo, 2008; Rubio et al., 2008), y en ambientes netamente boscosos y selváticos se agregan Tetragnathidae, Pholcidae y Linyphiidae destacándose entre las más abundantes (Russell-Smith \& Stork, 1994; Flórez-D., 1998, 1999; Sørensen, 2004; Benati et al., 2005). En los ambientes de las yungas se observaron las mismas familias dominantes, y en lo que respecta a Linyphiidae, Tetragnatidae, Pholcidae y Mysmenidae se observó su afinidad por la selva y el bosque montanos. Por otro lado el contraste del Chaco Serrano fue notable detectándose la afinidad de Oxyopidae sobre este ambiente y la dominancia relativa de Philodromidae, lo cual es coherente con otros estudios sobre araneofauna de pastizales y/o cultivos (Liljesthrom et al., 2002; Whitmore et al., 2002; Beltramo et al., 2006; Rodrigues et al., 2008; Rubio et al., 2008; Armendano \& Gonzalez, 2010).

La distribución rango/abundancia de especies en los ambientes de las yungas se ajustó a los modelos de distribución log-normal y serie logarítmica. Resultados similares se obtuvieron en estudios sobre la fauna de arañas en bosques tropicales y neotropicales cuyas distribuciones también fueron logarítmicas (e.g. Silva \& Coddington, 1996; Avalos et al., 2009). El ajuste a este tipo de distribución caracteriza a comunidades con bajo estrés y sometidas a la influencia de escasos factores (Moreno, 2001), representadas por pocas especies dominantes y pocas especies raras siendo la mayoría de abundancias intermedias (McNautghton \& Wolf, 1984; Magurran, 2004). Asimismo, una distribución log-normal es propia de las comunidades grandes (Magurran, 2004). Por otro lado, el Chaco Serrano fue la excepción para estas distribuciones reflejando sus mayores niveles de dominancia.

Al analizar dos facetas de la biodiversidad, a nivel local y regional, se obtiene una amplia perspectiva sobre las comunidades de arañas de las yungas. La diversidad $\beta$ mide las diferencias (el recambio) entre 
las especies de dos puntos, dos tipos de comunidades o dos paisajes (Halffter \& Moreno, 2005), y es el enlace entre las diversidades local y regional (Cornell $\&$ Lawton, 1992). En el contexto de diversidad $\beta$ en los distintos ambientes de yungas se observó que la proporción de especies compartidas fue baja, siendo la complementariedad superior al $60 \%$ entre la mayoría de los ambientes. Este valor relativamente alto es un indicativo de que las comunidades están suficientemente apartadas, mostrando una variación en las características de los ambientes y/o la estructura de su vegetación (Coddington et al., 1996). Por lo tanto, las excepciones que mostraron valores bajos de complementariedad (bosque montano vs. selva montana: $42 \%$, y selva pedemontana vs. yungas en transición: $58 \%$ ) podrían explicarse por relativas similitudes de los ambientes, o bien estar influenciadas por la distancia y contigüidad espacial (en parte, Fig. 1).

Las diferencias observadas en la composición de especies entre los distintos ambientes explican la significativa contribución de la diversidad $\beta$ a la riqueza de arañas. Resulta evidente que, a pesar de resaltar la importancia de la selva pedemontana y las yungas transicionales (como ambientes más diversos), la heterogeneidad espacial natural de toda la región también juega un papel relevante en la biodiversidad de estas arañas de vegetación en las selvas de yungas. En consecuencia, se sugiere que además de reforzar la protección en los pisos transicionales de yungas (hábitats más deteriorados), la gestión de conservación debería estar orientada en toda el área a promover la heterogeneidad espacial natural de las Yungas, haciendo especial hincapié en el mosaico de hábitats que constituyen cada estrato diferente.

El aporte de este trabajo sobre la fauna de arañas en la ecorregión de las Yungas argentinas es importante frente al escaso conocimiento previo. Así, se logra el primer estudio de biodiversidad de arañas del follaje integrado y asociado a estas selvas, mostrando no solo el alto valor en riqueza específica, sino también que géneros o especies de arañas se distribuyen diferencialmente según su afinidad o preferencia por los diferentes ambientes. Esta correspondencia con el tipo de ambiente fue verificada igualmente respecto de las comunidades que caracterizan a cada uno de ellos. También quedó en evidencia que la mayor similitud estructural entre ambientes tiene su correlato en la semejanza de sus comunidades de arañas de follaje.

\section{Agradecimientos}

Manifiesto mis agradecimientos a Luis E. Acosta y Alda González, quienes dirigieron mi tesis doctoral y contribuyeron en gran medida a este manuscrito derivado de dicha tesis. A Raquel M. Gleiser y Martín J. Ramírez por sus comentarios a versiones previas del manuscrito, y a Claudia E. Moreno por responder mis inquietudes acerca de los análisis de diversidad. A Carina I. Argañaraz y Martina Rubio por su valiosa colaboración en la depuración del material. Este trabajo fue financiado por la beca doctoral del CONICET.

\section{Referencias}

Acosta, L. E., 2002. Patrones zoogeográficos de los Opiliones argentinos (Arachnida: Opiliones). Revista Ibérica de Aracnología, 6: 69-84.

Armendano, A. \& González, A., 2010. Comunidad de arañas (Arachnida, Araneae) del cultivo de alfalfa en Buenos Aires, Argentina. International Journal of Tropical Biology, 58(2): 757-767. http://dx.doi.org/10.15517/rbt. v58i2.5243

Austin, M. P., 2002. Spatial prediction of species distribution: an interface between ecological theory and statistical modelling. Ecological Modelling, 157: 101-118. http:// dx.doi.org/10.1016/S0304-3800(02)00205-3

Avalos, G., Bar, M. E., Oscherov, E. B. \& González, A., 2013. Diversidad de Araneae en cultivos de Citrus sinensis (Rutaceae) de la Provincia de Corrientes, Argentina. International Journal of Tropical Biology, 61(3): 1243-1260. http://dx.doi.org/10.15517/rbt.v61i3.11938

Avalos, G., Damborsky, M. P., Bar, M. E., Oscherov, E. B. \& Porcel, E., 2009. Composición de la fauna de Araneae (Arachnida) de la Reserva provincial Iberá, Corrientes, Argentina. International Journal of Tropical Biology, 57(1-2): 339-351.

Avalos, G., Rubio, G. D., Bar, M. E. \& González, A., 2007. Arañas (Arachnida: Araneae) asociadas a dos bosques degradados del Chaco húmedo en Corrientes, Argentina. International Journal of Tropical Biology, 55(3-4): 899-909.

Baldissera, R., Rodrigues, E. N. L. \& Hartz, S. M., 2012. Metacommunity Composition of Web-Spiders in a Fragmented Neotropical Forest: Relative Importance of Environmental and Spatial Effects. PLoS ONE, 7(10): e48099 (9 pp.). http://dx.doi.org/10.1371/journal.pone. 0048099

Beltramo, J., Bertolaccini, I. \& González, A., 2006. Spiders of soybean crops in Santa Fe province, Argentina: influence of surrounding spontaneous vegetation on lot colonization. Brazilian Journal of Biology, 66(3): 891-898. http://dx. doi.org/10.1590/S1519-69842006000500015

Benati, K. R., Souza-Alves, J. P., Alves Silva, E., Lima Peres, M. C. \& Oliveira Coutinho, É., 2005. Aspectos comparativos das comunidades de aranhas (Araneae) em dois remanescentes de mata atlântica do estado da Bahia, Brasil. Biota Neotropica, 5(1a): 1-9. http://dx. doi.org/10.1590/S1676-06032005000200007

Bertonatti, C. \& Corcuera, J., 2000. Situación Ambiental argentina 2000. Fundación Vida Silvestre. Buenos Aires. 392 pp.

Blanco-Vargas, E., Amat-García, G. D. \& Flórez-Daza, E., 2003. Araneofauna orbitelar (Araneae: Orbiculariae) de los Andes de Colombia: comunidades en habitats bajo regeneración. Revista Ibérica de Aracnología, 7: 189-203.

Bonaldo, A. B. \& Dias, S. C., 2010. A structured inventory of spiders (Arachnida, Araneae) in natural and artificial forest gaps at Porto Urucu, Western Brazilian 
Amazonia. Acta Amazónica, 40(2): 357-372. http://dx. doi.org/10.1590/S0044-59672010000200014

Bonaldo, A. B., Marques, M. A. L., Pinto-da-Rocha, R. \& Gardner, T., 2007. Species richness and community structure of arboreal spider assemblages in fragments of three vegetational types at Banhado Grande wet plain, Gravataí River, Rio Grande do Sul, Brazil. Iheringia. Série Zoologia, 97(2): 143-151. http://dx.doi.org/10.1590/ S0073-47212007000200003

Brown, A. \& Malizia, L., 2004. Las selvas pedemontanas de las Yungas. Revista Ciencia Hoy (en línea), 14(83): 1-8.

Brown, A. D., Grau, A., Lomascolo, T. \& Gasparri, N., 2002. Estrategia de conservación para las selvas subtropicales de montaña (yungas) de argentina. Ecotrópicos, 15(2): 147-159.

Brown, A. D., Martínez Ortíz, U., Acerbi, M. \& Corcuera, J., 2006. La situación ambiental argentina 2005. Fundación Vida Silvestre Argentina. Buenos Aires. 587 pp.

Cabrera, A. L. \& Willink, A., 1973. Biogeografía de América Latina. OEA, Serie de Biología, Monografía 13, Washington D.C. 120 pp.

Cardoso, P., Scharff, N., Gaspar, C., Henriques, S. S., Carvalho, R., Castro, P. H., Schimdt, J. B., Silva, I., Szüts, T., de Castro, A. \& Crespo, L. C., 2008. Rapid biodiversity assessment of spiders (Araneae) using semiquantitative sampling: a case study in a Mediterranean forest. Insect Conservation and Diversity, 1: 71-84. http://dx.doi. org/10.1111/j.1752-4598.2007.00008.x

Carvalho, L. S. \& Avelino, M. T. L., 2010. Composição e diversidade da fauna de aranhas (Arachnida, Araneae) da Fazenda Nazareth, Município de José de Freitas, Piauí, Brasil. Biota Neotropica, 10(3): 1-11. http://dx. doi.org/10.1590/S1676-06032010000300001

Chao, A., Hwang, W., Chen, Y. \& Kuo, C., 2000. Estimating the number of shared species in two communities. Statistica Sinica, 10: 227-246.

Chebez, J. C., 2005. Guía de las reservas naturales de la Argentina: Noroeste. Albatros. 256 pp.

Clausen, I. H., 1986. The use of spiders (Araneae) as ecological indicators. Bulletin of the British Arachnological Society, 7: 83-86.

Coddington, J. A., Agnarsson, I., Miller, J. A., Kuntner, M. \& Hormiga, G., 2007. Undersampling bias: the null hypothesis for singleton species in tropical arthropod surveys. Abstracts of the 17 International Congress of Arachnology, São Pedro: 156.

Coddington, J. A. \& Levi, H., 1991. Systematics and evolution of spiders. Annual Review of Ecology and Systematics, 22: 111-128.

Coddington, J. A., Young, L. \& Coyle, F., 1996. Estimating spider species richness in a Southern Appalachian cove hardwood forest. Journal of Arachnology, 24: 111-128.

Colwell, R. K., 2006. EstimateS: Statistical estimation of species richness and shared species from samples. Version 8. Persistent URL < purl.oclc.org/estimates>

Colwell, R. K. \& Coddington, J., 1994. Estimating terrestrial biodiversity through extrapolation. Philosophical Transactions of the Royal Society, B: Biological Sciences, 345: 101-118. http://dx.doi.org/10.1098/rstb.1994.0091
Cornell, H. V. \& Lawton, J. H., 1992. Species interactions, local and regional processes, and limits to the richness of ecological communities: A teorical perspective. Journal of Animal Ecology, 61(1): 1-12. http://dx.doi. org $/ 10.2307 / 5503$

Cuezzo, F., Lizarralde De Grosso, M., Navarro, F. \& Szumik, C., 2007. Endemic insects from the Yungas of Argentina. Zootaxa, 1576: 63-67.

Diamond, J., 1988. Factors controlling species diversity: overview and synthesis. Annals of the Missouri Botanical Garden, 75(1): 117-119. http://dx.doi.org/10.2307/ 2399469

Dippenaar-Schoeman, A. S. \& Jocqué, R., 1997. African spiders an identification manual. Agricultural Research Council. Pretoria. 392 pp.

Enders, F., 1974. Vertical stratification in orb-web spiders (Araneidae, Araneae) and a consideration of other methods of coexistence. Ecology, 55(2): 317-328. http:// dx.doi.org/10.2307/1935219

Flórez-D, E., 1998. Estructura de comunidades de arañas (Araneae) en el departamento Del Valle, suroccidente de Colombia. Caldasia, 20: 173-192.

Flórez-D, E., 1999. Estudio de comunidades de arañas (Araneae) del Parque Nacional Farallones de Cali, Colombia. Cespedesia, 23(73-74): 99-113.

Grismado, C. J., Crudele, I., Damer, L., López, N., Olejnik, N. \& Trivero, S., 2011. Comunidades de arañas de la Reserva Natural Otamendi, provincia de Buenos Aires. Composición taxonómica y riqueza específica. Biológica, 14: 7-48.

Guisan, A. \& Zimmermann, N.E., 2000. Predictive habitat distribution model in ecology. Ecological Modelling, 135(2-3): 147-186. http://dx.doi.org/10.1016/S0304-3800 (00)00354-9

Gunnarsson, B., 1990. Vegetation structure and the abundance and size distribution of spruce-living spiders. Journal of animal Ecology, 59(2): 743-752. http://dx. doi.org/10.2307/4892

Halffter, G. \& Moreno, C.E., 2005. Significado biológico de las diversidades Alfa, Beta y Gamma. In: G. Halffter, J. Soberón, P. Koleff \& A. Melic (eds.). Sobre Diversidad Biológica: El significado de las Diversidades Alfa, Beta y Gamma. Monografías Tercer Milenio, S.E.A. Zaragoza: 5-18.

Hammer, O., Harper, D. A. T. \& Ryan, P. D., 2003. PAST: Palaeontological Statistics, Version 1.18. Available at: http://folk.uio.no/ohammer/past

Hartmut, G. D., Denno, R. F. \& Coddington, J. A., 1990. Spider (Araneae) community structure in an intertidial salt marsh: efects of vegetation structure and tidal flooding. Environmental Entomology, 19(5): 1356-1370.

Hatley, C. \& MacMahom, J., 1980. Spider community organization: Seasonal variation and the role of vegetation architecture. Environmental Entomology, 9: 632-639. $\mathrm{http}: / / d x . d o i . o r g / 10.1093 / \mathrm{ee} / 9.5 .632$

Höfer, H., 1990. The spider community (Araneae) of a Central Amazonian blackwater inundation forest (Igapó). Acta Zoologica Fennica, 190: 173-179.

Humphries, C. J., Williams, P. H. \& Vane-Wright, R. I., 1995. Measuring biodiversity value for conservation. 
Annual Review of Ecology and Systematics, 26: 93-111. http://dx.doi.org/10.1146/annurev.es.26.110195.000521

Janzen, D., Ataroff, M., Farinas, M., Reyes, S., Rincon, N., Soler, A., Soriano, P. \& Vera, M., 1976. Changes in the arthropod community along an elevational transect in the Venezuelan Andes. Biotropica, 8(3): 193-203. http:// dx.doi.org/10.2307/2989685

Jiménez-Valverde, A. \& Lobo, J.M., 2007. Determinants of local spider (Araneidae and Thomisidae) species richness on a regional scale: climate and altitude vs. habitat structure. Ecological Entomology, 32: 113-122. http:// dx.doi.org/10.1111/j.1365-2311.2006.00848.x

Jongman, G., Ter Braak, C. \& Van Tongeren, O., 1995. Data analysis in community and landscape ecology. Cambridge University Press. 299 pp.

Krebs, C. J., 1989. Ecological methodology. Black Well Scientific Press. 654 pp.

Labarque, F. M., Piacentini, L. N., Ramírez, M. J., Hormiga, G., Dimitrov, D., Benavides, L., Arnedo, M. A. \& Pons, J., 2010. PANCODING: estimando patrones de biodiversidad utilizando códigos de barras del ADN. Resúmenes de la IX Reunión Argentina de Cladística y Biogeografia, La Plata: 78.

LIEY \& FVSA., 2000. Descripción y manejo de posibles nuevas áreas naturales protegidas en las yungas de la Argentina. Fundación Vida Silvestre. 21 pp.

Liljesthröm, G., Minervino, E., Castro, D. \& Gonzalez, A., 2002. La comunidad de arañas del cultivo de soja en la provincia de Buenos Aires, Argentina. Neotropical Entomology, 31(2): 197-210. http://dx.doi.org/10.1590/ S1519-566X2002000200005

Machado Pinto-Leite, C., Camacho Guerrero, A. \& Kobler Brazil, T., 2008. Non-random patterns of spider species composition in an Atlantic rainforest. Journal of Arachnology, 36(2): 448-452. http://dx.doi.org/10.1636/ CT07-123.1

Maelfert, J., Jocqué, R., Baert, L. \& Descender, K., 1990. Heathland management and spiders. Acta Zoologica Fennica, 190: 261-266.

Magurran, A. E., 2004. Measuring biological diversity. Blackwell Science. Oxford. 256 pp.

McAleece, N., 1999. BioDiversity PRO. Ver. 2.0.0. The Natural History Museum \& The Scottish Association for Marine Science.

McCoy, E. D., 1990. The distribution of insects along elevational gradients. Oikos, 58(3): 313-322. http://dx. doi.org/10.2307/3545222

McNautghton, S. J. \& Wolf, L. L., 1984. Ecología General. Omega. Barcelona. 713 pp.

Meyke, E., 1999-2004. TAXIS 3.5-Taxonomical Information System. www.bio-tools-net

Moreno, C. E., 2001. Métodos para medir la biodiversidad. M\&T - Manuales y Tesis SEA, vol. 1. Zaragoza. 84 pp.

Morrone, J. J., 2006. Biogeographic areas and transition zones of Latin America and the Caribbean Islands based on analyses of the entomofauna. Annual Review of Entomology, 51(1): 467-94. http://dx.doi.org/10.1146/ annurev.ento.50.071803.130447

Navarro, F. R., Cuezzo, F., Goloboff, P. A., Szumik, C., Lizarralde De Grosso, M. \& Quintana, M. G., 2009.
Can insect data be used to infer areas of endemism? An example from the Yungas of Argentina. Revista Chilena de Historia Natural, 82(4): 507-522. http://dx.doi.org/ 10.4067/S0716-078X2009000400006

Nogueira, A. A., Pinto-da-Rocha, R. \& Brescovit, A. D., 2006. Comunidade de aranhas orbítelas (Araneae, Arachnida) na região da Reserva Florestal do Morro Grande, Cotia, São Paulo, Brasil. Biota Neotropica, 6(2): 1-24. http:// dx.doi.org/10.1590/S1676-06032006000200010

Oliver, I. \& Beattie, A. J., 1993. A possible method for the rapid assessment of biodiversity. Conservation Biology, 7(3): 562-568. http://dx.doi.org/10.1046/j.1523-1739. 1993.07030562.x

Olson, D. M., Dinerstein, E., Wikramanayake, E. D., Burgess, N. D., Powell, G. V. N., Underwood, E. C., D’Amico, J. A., Strand, H. E., Morrison, J. C., Loucks, C. J., Allnutt, T. F., Lamoreux, J. F., Ricketts, T. H., Itoua, I., Wettengel, W. W., Kura, Y., Hedao, P. \& Kassem, K., 2001. Terrestrial ecoregions of the world: a new map of life on Earth. BioScience, 51: 933-938. http:// dx.doi.org/10.1641/0006-3568(2001)051\%5B0933: TEOTWA $\% 5 \mathrm{D} 2.0 . \mathrm{CO} ; 2$

Pérez-Miles, F., Simó, M., Toscano-Gadea, C. \& Useta, G., 1999. La comunidad de Araneae criptozóicas del Cerro de Montevideo, Uruguay: Un ambiente rodeado por urbanización. Physis, C57: 73-78.

Pinkus-Rendón, M. A., León-Cortés, J. L. \& Ibarra-Nuñez, G., 2006. Spiders diversity in a tropical habitat gradient in Chiapas, Mexico. Diversity and Distributions, 12: 61-69. http://dx.doi.org/10.1111/j.1366-9516.2006.00217.x

Podgaiski, L.R., Ott, R., Lopes Rodrigues, E.N., Buckup, E.H. \& Leão Marques, M.A., 2007. Araneofauna (Arachnida; Araneae) do Parque Estadual do Turvo, Rio Grande do Sul, Brasil. Biota Neotropica, 7(2): 1-15.

Raizer, J., Japyassú, H.F., Indicatti, R.P. \& Brescovit, A.D., 2005. Comunidade de aranhas (Arachnida, Araneae) do Pantanal Norte (Mato Grosso, Brasil) e sua similaridade com a araneofauna amazônica. Biota Neotropica, 5(1): 1-16. http://dx.doi.org/10.1590/S167606032007000200023

Ramírez, M.J., Labarque, F. M., Piacentini, L. N., Hormiga, G., Dimitrov, D., Benavides, L., Arnedo, M. A. \& Pons, J., 2010. PANCODING: estimaciones comparadas de efíciencia en la determinación de inventarios de biodiversidad utilizando combinaciones de morfología y códigos de barras del ADN. Resúmenes de la IX Reunión Argentina de Cladística y Biogeografía, La Plata: 99.

Ricetti, J. \& Bonaldo, A.B., 2008. Diversidade e estimativas de riqueza de aranhas em quatro fitofisionomias na Serra do Cachimbo, Pará, Brasil. Iheringia. Série Zoologia, 98(1): 88-99. http://dx.doi.org/10.1590/S007347212008000100013

Rodrigues, E. N. L., 2005. Fauna araneológica (Arachnida; Araneae) arborícola de duas áreas em uma mata de restinga no sul do Brasil. Acta Biológica Leopoldensia, 27(1): 73-92.

Rodrigues, E. N. L., Mendonça, M. S. Jr. \& Ott, R., 2008. Fauna de aranhas (Arachnida, Araneae) em diferentes estágios do cultivo do arroz irrigado em Cachoeirinha, RS, Brasil. Iheringia. Série Zoologia, 98(3): 362-371. http://dx.doi.org/10.1590/S0073-47212008000300011 
Rubio, G. D., 2014. Baseline richness of Salticidae (Araneae) from Misiones, Argentina. Peckhamia, 118.1: 1-21.

Rubio, G. D., Corronca, J. A. \& Damborsky, M. P., 2008. Do spider diversity and assemblages change in different contiguous habitats? A case study in the protected habitats of the Humid Chaco ecoregion, north-east Argentina. Environmental Entomology, 37: 419-430.

Rubio, G. D. \& Moreno, C. E., 2010. Orb-weaving spider diversity in the Iberá marshlands (Argentina). Neotropical Entomology, 39(4): 496-505. http://dx.doi.org/10.1590/ S1519-566X2010000400006

Russell-Smith, A. \& Stork, N. E., 1994. Abundance and diversity of spiders from the canopy of tropical rainforests with particular reference to Sulawesi, Indonesia. Journal of Tropical Ecology, 10(4): 545-558. http://dx. doi.org/10.1017/S0266467400008221

Silva, D. \& Coddington, J. A., 1996. Spiders of Pakitza (Madre de Dios, Peru): species richness y notes in community structure. In: D.E. Wilson \& A. Sandoval (eds.). The biodiversity of Pakitza y its environs. Smithsonian Institution. Washington: 241-299.

Sørensen, L. L., 2004. Composition and diversity of the spider fauna in the canopy of a montane forest in Tanzania. Biodiversity and Conservation, 13: 437-452. http://dx. doi.org/10.1023/B:BIOC.0000006510.49496.1e

Sørensen, L. L., Coddington, J. A. \& Scharff, N., 2002. Inventorying and estimating sub-canopy spider diversity using semi-quantitative sampling methods in an
Afromontane forest. Environmental Entomology, 31: 319-330

Toti, D., Coyle, F. \& Miller, J., 2000. A structured inventory of Appalachian grass Bald and heath Bald spider assemblages and a test of species richness estimator performance.Journal of Arachnology, 28:329-345.http:// dx.doi.org/10.1636/0161-8202(2000)028\%5B0329: ASIOAG\%5D2.0.CO;2

Turnbull, A. L., 1973. Ecology of the true spiders (Araenomorpha). Annual Review of Entomology, 18: 305-348.

Uetz, G. W., 1976. Gradient analisis of spiders communities in a streamside forest. Oecologia, 22: 373-385. http:// dx.doi.org/10.1007/BF00345314

Uetz, G. W., Halaj, J. \& Cady, A. B., 1999. Guild structure of spiders in major crops. Journal of Arachnology, 27: 270-280.

Whitmore, C., Slotow, R., Crouch, T. E. \& DippenaarSchoeman, A. S., 2002. Diversity of spiders (Araneae) in a savanna reserve, Northern Province, South Africa. Journal of Arachnology, 30: 344-356. http://dx.doi. org/10.1636/0161-8202(2002)030\%5B0344:DO SAIA $\% 5 \mathrm{D} 2.0 . \mathrm{CO} ; 2$

Wise, D. H., 1993. Spider in ecological webs. Cambridge University Press. Cambridge. 328 pp. http://dx.doi.org/ $10.1017 / \mathrm{CBO} 9780511623431$

Wolda, H., 1987. Altitude, habitat and tropical insect diversity. Biological Journal of the Linnean Society, 30: 313-323. http://dx.doi.org/10.1111/j.1095-8312.1987.tb00305.x 
Apéndice 1.- Familias, especies y abundancia de arañas en los diferentes ambientes de las yungas de Salta, Argentina. En negrita se indican las especies dominantes. La última columna muestra los valores del ChS a modo comparativo.

Appendix 1.- Families, species and abundance of spiders in the different environments of the Yungas of Salta, Argentina. In bold are the dominant species. The last column shows the ChS values for comparison.

\begin{tabular}{|c|c|c|c|c|c|c|c|c|}
\hline Familia & Determinación & Cc_s & YT & SP & SM & BM & $\begin{array}{c}\text { Total } \\
\text { Yungas }\end{array}$ & ChS \\
\hline Amaurobiidae & Ama_msp240 & 0 & 0 & 2 & 0 & 0 & 2 & 0 \\
\hline \multirow[t]{2}{*}{ Anapidae } & Ana_msp233 & 0 & 0 & 0 & 1 & 1 & 2 & 0 \\
\hline & Ana_msp241 & 0 & 0 & 1 & 0 & 0 & 1 & 0 \\
\hline \multirow[t]{14}{*}{ Anyphaenidae } & cf_Temnida_msp131 & 30 & 142 & 106 & 40 & 47 & 365 & 333 \\
\hline & Josa_msp126 & 0 & 0 & 1 & 53 & 46 & 100 & 0 \\
\hline & Sanogasta_msp190 & 0 & 92 & 1 & 0 & 0 & 93 & 1 \\
\hline & Any_msp36 & 10 & 40 & 23 & 9 & 5 & 87 & 3 \\
\hline & Any_msp145 & 11 & 23 & 24 & 5 & 3 & 66 & 0 \\
\hline & Tasata_msp112 & 0 & 0 & 2 & 15 & 17 & 34 & 0 \\
\hline & Arachosia_msp74 & 0 & 5 & 0 & 16 & 10 & 31 & 4 \\
\hline & Negayan_msp133 & 0 & 8 & 2 & 0 & 2 & 12 & 0 \\
\hline & cf_Josa_msp174 & 2 & 0 & 0 & 6 & 4 & 12 & 0 \\
\hline & Any_msp147 & 1 & 0 & 1 & 3 & 0 & 5 & 0 \\
\hline & Teudis_msp93 & 0 & 1 & 1 & 1 & 0 & 3 & 0 \\
\hline & cf_Temnida_msp209 & 0 & 0 & 1 & 0 & 1 & 2 & 0 \\
\hline & Teudis_msp239 & 0 & 0 & 1 & 0 & 0 & 1 & 0 \\
\hline & Teudis_msp250 & 0 & 1 & 0 & 0 & 0 & 1 & 3 \\
\hline \multirow[t]{30}{*}{ Araneidae } & Mangora cochuna Levi, 2007 & 0 & 0 & 2 & 69 & 31 & 102 & 0 \\
\hline & Ara_msp230 & 0 & 2 & 0 & 55 & 19 & 76 & 0 \\
\hline & Araneus_msp69 & 8 & 46 & 4 & 3 & 2 & 63 & 36 \\
\hline & Metazygia viriosa (Keyserling, 1892) & 11 & 17 & 1 & 20 & 11 & 60 & 90 \\
\hline & Ocrepeira_msp43 & 0 & 39 & 3 & 0 & 0 & 42 & 6 \\
\hline & Ocrepeira_msp60 & 3 & 10 & 11 & 4 & 0 & 28 & 13 \\
\hline & Micrathena shealsi Chickering, 1960 & 0 & 0 & 0 & 13 & 11 & 24 & 0 \\
\hline & Micrathena_msp59 & 0 & 1 & 5 & 5 & 13 & 24 & 0 \\
\hline & Ara_msp135 & 5 & 12 & 0 & 0 & 0 & 17 & 11 \\
\hline & Mecynogea erythromela (Holmberg, 1876) & 8 & 7 & 0 & 0 & 0 & 15 & 1 \\
\hline & Ara_msp103 & 1 & 7 & 1 & 3 & 0 & 12 & 1 \\
\hline & Mangora v-signata Mello-Leitão, 1943 & 0 & 7 & 2 & 0 & 0 & 9 & 0 \\
\hline & Parawixia audax (Blackwall, 1863) & 0 & 4 & 3 & 0 & 0 & 7 & 3 \\
\hline & Alpaida_msp189 & 2 & 4 & 0 & 0 & 0 & 6 & 6 \\
\hline & Ara_msp137 & 0 & 2 & 0 & 3 & 0 & 5 & 0 \\
\hline & Ara_msp149 & 0 & 0 & 5 & 0 & 0 & 5 & 0 \\
\hline & Alpaida_msp100 & 0 & 0 & 4 & 0 & 0 & 4 & 0 \\
\hline & Metepeira_msp194 & 1 & 3 & 0 & 0 & 0 & 4 & 2 \\
\hline & Cyclosa_msp195 & 2 & 2 & 0 & 0 & 0 & 4 & 6 \\
\hline & Ocrepeira_msp199 & 2 & 2 & 0 & 0 & 0 & 4 & 32 \\
\hline & Mangora cf chuquisacai Levi, 2007 & 0 & 0 & 0 & 0 & 3 & 3 & 0 \\
\hline & Ara_msp153 & 0 & 2 & 1 & 0 & 0 & 3 & 0 \\
\hline & Micrathena crassa (Keyserling, 1864) & 0 & 0 & 0 & 0 & 3 & 3 & 0 \\
\hline & Wagneriana madrejon Levi, 1991 & 0 & 3 & 0 & 0 & 0 & 3 & 1 \\
\hline & Ara_msp132 & 0 & 1 & 1 & 0 & 0 & 2 & 0 \\
\hline & Argiope argentata (Fabricius, 1775) & 0 & 2 & 0 & 0 & 0 & 2 & 3 \\
\hline & Mastophora_msp203 & 1 & 1 & 0 & 0 & 0 & 2 & 5 \\
\hline & Ara_msp157 & 1 & 0 & 0 & 0 & 0 & 1 & 2 \\
\hline & Ara_msp2 & 0 & 0 & 0 & 0 & 1 & 1 & 0 \\
\hline & Kaira_msp221 & 1 & 0 & 0 & 0 & 0 & 1 & 2 \\
\hline
\end{tabular}


Appendix 1.- (Continued)

\begin{tabular}{|c|c|c|c|c|c|c|c|c|}
\hline Familia & Determinación & Cc_s & YT & SP & SM & BM & $\begin{array}{c}\text { Total } \\
\text { Yungas }\end{array}$ & ChS \\
\hline & Micrathena_msp224 & 0 & 1 & 0 & 0 & 0 & 1 & 1 \\
\hline & Acacesia_msp244 & 0 & 0 & 0 & 0 & 1 & 1 & 0 \\
\hline & cf_Larinia_msp246 & 0 & 1 & 0 & 0 & 0 & 1 & 0 \\
\hline & cf_Araneus_msp32 & 1 & 0 & 0 & 0 & 0 & 1 & 0 \\
\hline \multirow[t]{7}{*}{ Corinnidae } & Castianeirinae_msp188 & 0 & 6 & 2 & 0 & 9 & 17 & 1 \\
\hline & Cor_msp235 & 0 & 0 & 5 & 1 & 0 & 6 & 2 \\
\hline & Cor_msp71 & 6 & 0 & 0 & 0 & 0 & 6 & 22 \\
\hline & Castianeirinae_msp236 & 0 & 2 & 2 & 0 & 0 & 4 & 0 \\
\hline & cf_Meriola_msp180 & 0 & 1 & 1 & 0 & 1 & 3 & 0 \\
\hline & Castianeira_msp198 & 0 & 1 & 1 & 0 & 0 & 2 & 2 \\
\hline & Meriola_msp255 & 0 & 0 & 1 & 0 & 0 & 1 & 0 \\
\hline Ctenidae & Cte_msp237 & 0 & 2 & 1 & 0 & 0 & 3 & 0 \\
\hline \multirow[t]{2}{*}{ Dictynidae } & Dictyna_msp150 & 5 & 3 & 8 & 0 & 0 & 16 & 0 \\
\hline & Dictyna_msp171 & 7 & 3 & 0 & 0 & 0 & 10 & 0 \\
\hline Diguetidae & Diguetia catamarquensis (Mello-Leitão, 1941) & 1 & 0 & 0 & 0 & 0 & 1 & 0 \\
\hline Filistatidae & Fil_msp223 & 2 & 0 & 0 & 0 & 0 & 2 & 0 \\
\hline \multirow[t]{4}{*}{ Gnaphosidae } & Gna_msp138 & 1 & 1 & 1 & 0 & 0 & 3 & 0 \\
\hline & Gna_msp181 & 0 & 2 & 0 & 0 & 1 & 3 & 0 \\
\hline & Gna_msp177 & 1 & 0 & 0 & 0 & 1 & 2 & 0 \\
\hline & Gna_msp173 & 0 & 0 & 1 & 0 & 0 & 1 & 0 \\
\hline Hahniidae & Hahnia_msp115 & 0 & 0 & 0 & 10 & 6 & 16 & 0 \\
\hline \multirow[t]{9}{*}{ Linyphiidae } & Dubiaranea_msp111 & 1 & 5 & 36 & 192 & 187 & 421 & 1 \\
\hline & Dubiaranea difficilis (Mello-Leitão, 1944) & 1 & 16 & 21 & 135 & 88 & 261 & 2 \\
\hline & cf_Maro_msp108 & 0 & 3 & 3 & 38 & 6 & 50 & 0 \\
\hline & Lin_msp213 & 0 & 0 & 0 & 7 & 4 & 11 & 0 \\
\hline & Lin_msp28 & 1 & 1 & 0 & 0 & 3 & 5 & 1 \\
\hline & Lin_msp178 & 0 & 0 & 0 & 0 & 4 & 4 & 0 \\
\hline & Agyneta_msp96 & 0 & 0 & 1 & 2 & 0 & 3 & 1 \\
\hline & Dubiaranea_msp245 & 0 & 0 & 0 & 1 & 0 & 1 & 0 \\
\hline & Lin_msp86 & 0 & 1 & 0 & 0 & 0 & 1 & 0 \\
\hline \multirow[t]{4}{*}{ Lycosidae } & Lobizon ojangureni Piacentini \& Grismado, 2009 & 0 & 1 & 1 & 20 & 23 & 45 & 0 \\
\hline & Aglaoctenus_msp205 & 1 & 2 & 0 & 0 & 0 & 3 & 4 \\
\hline & Sossipinae_msp229 & 0 & 0 & 0 & 0 & 1 & 1 & 0 \\
\hline & Lyc_msp7 & 0 & 0 & 0 & 0 & 1 & 1 & 0 \\
\hline \multirow[t]{2}{*}{ Mimetidae } & cf_Mimetus_msp215 & 2 & 1 & 0 & 13 & 5 & 21 & 0 \\
\hline & Gelanor_cf_zonatus & 0 & 1 & 13 & 0 & 0 & 14 & 9 \\
\hline \multirow[t]{2}{*}{ Miturgidae } & cf_Eutichurus_msp119 & 0 & 5 & 2 & 2 & 3 & 12 & 0 \\
\hline & Mit_msp78 & 1 & 0 & 0 & 0 & 0 & 1 & 0 \\
\hline \multirow[t]{2}{*}{ Mysmenidae } & Mysmena_msp110 & 0 & 1 & 9 & 170 & 230 & 410 & 3 \\
\hline & Trogloneta_msp146 & 0 & 0 & 0 & 7 & 6 & 13 & 0 \\
\hline Nephilidae & Nephila clavipes (Linnaeus, 1767) & 0 & 32 & 2 & 0 & 0 & 34 & 0 \\
\hline Oonopidae & Oonops tucumanus Simon, 1907 & 0 & 0 & 1 & 0 & 0 & 1 & 0 \\
\hline \multirow[t]{4}{*}{ Oxyopidae } & Oxy_msp50 & 6 & 17 & 3 & 0 & 0 & 26 & 1 \\
\hline & Peucetia rubrolineata Keyserling, 1877 & 2 & 0 & 9 & 0 & 0 & 11 & 1 \\
\hline & Oxyopes_msp11 & 0 & 4 & 5 & 0 & 0 & 9 & 47 \\
\hline & Oxy_msp257 & 0 & 1 & 0 & 0 & 0 & 1 & 2 \\
\hline \multirow[t]{3}{*}{ Philodromidae } & Paracleocnemis_msp75 & 45 & 86 & 3 & 0 & 0 & 134 & 133 \\
\hline & Phi_msp226 & 2 & 1 & 0 & 0 & 0 & 3 & 0 \\
\hline & Phi_msp225 & 0 & 1 & 0 & 0 & 0 & 1 & 0 \\
\hline
\end{tabular}


Appendix 1.- (Continued)

\begin{tabular}{|c|c|c|c|c|c|c|c|c|}
\hline Familia & Determinación & Cc_s & YT & SP & SM & BM & $\begin{array}{c}\text { Total } \\
\text { Yungas }\end{array}$ & ChS \\
\hline \multirow[t]{3}{*}{ Pholcidae } & Chibchea salta Huber, 2000 & 2 & 4 & 0 & 262 & 250 & 518 & 0 \\
\hline & Pho_msp113 & 4 & 0 & 0 & 1 & 0 & 5 & 0 \\
\hline & Pho_msp258 & 0 & 3 & 0 & 0 & 0 & 3 & 0 \\
\hline Pisairidae & Pis_msp124 & 0 & 0 & 3 & 2 & 0 & 5 & 0 \\
\hline \multirow[t]{21}{*}{ Salticidae } & cf_Mopiopia_msp94 & 2 & 2 & 1 & 115 & 23 & 143 & 0 \\
\hline & Sal_msp183 & 0 & 0 & 0 & 11 & 54 & 65 & 0 \\
\hline & cf_Sassacus_msp136 & 1 & 16 & 17 & 3 & 5 & 42 & 0 \\
\hline & Sal_msp16 & 2 & 9 & 21 & 4 & 1 & 37 & 1 \\
\hline & Lyssomanes_msp18 & 0 & 0 & 35 & 0 & 0 & 35 & 0 \\
\hline & Sal_msp31 & 4 & 26 & 0 & 0 & 0 & 30 & 17 \\
\hline & Sal_msp123 & 1 & 11 & 5 & 5 & 5 & 27 & 0 \\
\hline & Sal_msp12 & 4 & 18 & 3 & 1 & 0 & 26 & 0 \\
\hline & Sal_msp63 & 1 & 7 & 11 & 0 & 0 & 19 & 2 \\
\hline & Sal_msp20 & 2 & 12 & 0 & 1 & 1 & 16 & 3 \\
\hline & Sal_msp207 & 2 & 11 & 1 & 0 & 0 & 14 & 14 \\
\hline & Sal_msp95 & 0 & 0 & 14 & 0 & 0 & 14 & 5 \\
\hline & Sal_msp220 & 1 & 4 & 5 & 0 & 0 & 10 & 0 \\
\hline & Sal_msp83 & 5 & 3 & 2 & 0 & 0 & 10 & 1 \\
\hline & Sal_msp219 & 0 & 7 & 1 & 1 & 0 & 9 & 0 \\
\hline & Sal_msp251 & 0 & 5 & 1 & 0 & 0 & 6 & 0 \\
\hline & Sal_msp134 & 0 & 2 & 0 & 0 & 2 & 4 & 0 \\
\hline & Sal_msp249 & 2 & 1 & 0 & 0 & 0 & 3 & 0 \\
\hline & Sal_msp44 & 0 & 2 & 0 & 0 & 1 & 3 & 9 \\
\hline & Sal_msp248 & 0 & 2 & 0 & 0 & 0 & 2 & 0 \\
\hline & Sarinda_msp45 & 0 & 0 & 1 & 0 & 1 & 2 & 0 \\
\hline Scytodidae & Scytodes_msp159 & 4 & 3 & 0 & 0 & 0 & 7 & 0 \\
\hline \multirow[t]{3}{*}{ Sparassidae } & Spa_msp152 & 2 & 0 & 9 & 0 & 0 & 11 & 1 \\
\hline & cf_Olios_msp206 & 0 & 4 & 3 & 0 & 0 & 7 & 3 \\
\hline & Spa_msp89 & 1 & 0 & 0 & 0 & 0 & 1 & 0 \\
\hline \multirow[t]{2}{*}{ Symphytognathidae } & cf_Globignatha_msp184 & 0 & 0 & 0 & 2 & 3 & 5 & 0 \\
\hline & Anapistula yungas Rubio \& González, 2010 & 0 & 0 & 0 & 2 & 0 & 2 & 0 \\
\hline \multirow[t]{7}{*}{ Tetragnathidae } & Leucauge cf venusta (Walckenaer, 1842) & 0 & 37 & 37 & 91 & 122 & 287 & 31 \\
\hline & cf_Chrysometa_msp187 & 0 & 0 & 0 & 111 & 175 & 286 & 0 \\
\hline & Tet_msp117 & 0 & 1 & 0 & 30 & 32 & 63 & 0 \\
\hline & Tet_msp57 & 0 & 48 & 1 & 2 & 1 & 52 & 1 \\
\hline & Tet_msp102 & 2 & 1 & 1 & 6 & 6 & 16 & 0 \\
\hline & cf_Chrysometa_msp217 & 0 & 8 & 1 & 6 & 0 & 15 & 1 \\
\hline & Tet_msp232 & 0 & 0 & 0 & 0 & 4 & 4 & 0 \\
\hline \multirow[t]{11}{*}{ Theridiidae } & The_msp47 & 3 & 26 & 79 & 75 & 42 & 225 & 18 \\
\hline & Anelosimus_msp118 & 0 & 2 & 0 & 130 & 4 & 136 & 0 \\
\hline & The_msp109 & 0 & 0 & 61 & 38 & 23 & 122 & 0 \\
\hline & The_msp122 & 0 & 0 & 22 & 53 & 43 & 118 & 0 \\
\hline & The_msp52 & 5 & 8 & 6 & 32 & 36 & 87 & 2 \\
\hline & The_msp125 & 0 & 4 & 4 & 22 & 56 & 86 & 0 \\
\hline & The_msp161 & 46 & 1 & 0 & 0 & 0 & 47 & 0 \\
\hline & The_msp58 & 2 & 19 & 14 & 3 & 3 & 41 & 29 \\
\hline & The_msp91 & 0 & 0 & 34 & 2 & 1 & 37 & 0 \\
\hline & Argyrodes_msp192 & 3 & 22 & 1 & 0 & 0 & 26 & 2 \\
\hline & The_msp51 & 0 & 0 & 5 & 20 & 1 & 26 & 0 \\
\hline
\end{tabular}


Appendix 1.- (Continued)

\begin{tabular}{|c|c|c|c|c|c|c|c|c|}
\hline Familia & Determinación & Cc_s & YT & SP & SM & BM & $\begin{array}{c}\text { Total } \\
\text { Yungas }\end{array}$ & ChS \\
\hline & Argyrodes_msp162 & 6 & 7 & 0 & 4 & 3 & 20 & 2 \\
\hline & The_msp98 & 0 & 0 & 3 & 12 & 5 & 20 & 0 \\
\hline & The_msp37 & 0 & 2 & 1 & 3 & 8 & 14 & 0 \\
\hline & The_msp142 & 0 & 0 & 0 & 3 & 10 & 13 & 0 \\
\hline & The_msp231 & 0 & 0 & 0 & 9 & 4 & 13 & 0 \\
\hline & Spintharus_msp210 & 0 & 0 & 0 & 2 & 6 & 8 & 0 \\
\hline & The_msp218 & 0 & 7 & 0 & 1 & 0 & 8 & 0 \\
\hline & The_msp99 & 0 & 0 & 0 & 6 & 1 & 7 & 0 \\
\hline & The_msp243 & 0 & 0 & 0 & 0 & 5 & 5 & 0 \\
\hline & Argyrodes_msp164 & 1 & 2 & 0 & 1 & 0 & 4 & 0 \\
\hline & The_msp167 & 2 & 0 & 0 & 0 & 2 & 4 & 0 \\
\hline & Dipoena_msp256 & 0 & 0 & 4 & 0 & 0 & 4 & 0 \\
\hline & The_msp155 & 0 & 0 & 1 & 1 & 1 & 3 & 0 \\
\hline & Ariamnes cf longissimus (Keyserling, 1891) & 0 & 3 & 0 & 0 & 0 & 3 & 0 \\
\hline & The_msp70 & 0 & 0 & 0 & 3 & 0 & 3 & 0 \\
\hline & Euryopis_msp186 & 0 & 0 & 0 & 1 & 1 & 2 & 0 \\
\hline & Argyrodes_msp242 & 0 & 0 & 0 & 1 & 1 & 2 & 0 \\
\hline & The_msp204 & 0 & 0 & 1 & 0 & 0 & 1 & 11 \\
\hline & Euryopis_msp216 & 0 & 0 & 0 & 0 & 1 & 1 & 0 \\
\hline & Phoroncidia cribrata (Simon, 1893) & 0 & 1 & 0 & 0 & 0 & 1 & 0 \\
\hline \multirow[t]{5}{*}{ Theridiosomatidae } & Theridiosoma_msp140 & 0 & 0 & 0 & 7 & 23 & 30 & 0 \\
\hline & Theso_msp127 & 0 & 0 & 1 & 2 & 4 & 7 & 0 \\
\hline & cf_Ogulnius_msp84 & 0 & 1 & 4 & 0 & 2 & 7 & 0 \\
\hline & Theso_msp185 & 0 & 0 & 2 & 0 & 0 & 2 & 0 \\
\hline & Chthonos_msp197 & 0 & 2 & 0 & 0 & 0 & 2 & 0 \\
\hline \multirow[t]{12}{*}{ Thomisidae } & cf_Misumenops_msp27 & 76 & 157 & 55 & 2 & 1 & 291 & 81 \\
\hline & Tmarus estyliferus Mello-Leitão, 1929 & 5 & 61 & 16 & 1 & 0 & 83 & 0 \\
\hline & Tmarus_msp54 & 5 & 29 & 13 & 0 & 0 & 47 & 22 \\
\hline & Onocolus_msp121 & 0 & 0 & 0 & 8 & 3 & 11 & 0 \\
\hline & Tho_msp238 & 0 & 0 & 11 & 0 & 0 & 11 & 0 \\
\hline & Tho_msp214 & 0 & 0 & 0 & 7 & 1 & 8 & 0 \\
\hline & Tho_msp191 & 0 & 4 & 0 & 0 & 0 & 4 & 1 \\
\hline & Tho_msp222 & 1 & 2 & 0 & 0 & 0 & 3 & 0 \\
\hline & Tho_msp65 & 1 & 0 & 1 & 0 & 1 & 3 & 0 \\
\hline & cf_Misumenoides_msp211 & 1 & 0 & 0 & 0 & 1 & 2 & 0 \\
\hline & Sidymella_msp64 & 0 & 1 & 0 & 1 & 0 & 2 & 0 \\
\hline & Tho_msp254 & 0 & 1 & 0 & 0 & 0 & 1 & 0 \\
\hline Titanoecidae & Goeldia_msp49 & 0 & 0 & 2 & 0 & 0 & 2 & 0 \\
\hline Trechaleidae & Dossenus_msp227 & 0 & 0 & 3 & 0 & 0 & 3 & 0 \\
\hline \multirow[t]{3}{*}{ Uloboridae } & Uloborus_msp154 & 1 & 0 & 20 & 0 & 1 & 22 & 2 \\
\hline & Philoponella_msp160 & 13 & 0 & 1 & 0 & 0 & 14 & 2 \\
\hline & Miagrammopes_msp151 & 0 & 3 & 2 & 0 & 0 & 5 & 0 \\
\hline Zodariidae & Zod_msp141 & 0 & 1 & 0 & 13 & 0 & 14 & 1 \\
\hline 34 Familias & 188 Especies & 399 & 1301 & 881 & 2041 & 1790 & 6412 & 1059 \\
\hline
\end{tabular}

